SPOR-Report 2000-11

\title{
A Class of Distribution-free Control Charts
}

S. Chakraborti

P. van der Laan

M.A. van de Wiel 


\title{
A Class of Distribution-free Control Charts ${ }^{1}$
}

\author{
S. Chakraborti \\ Applied Statistics Program \\ Department of Management Science and Statistics \\ University of Alabama \\ Tuscaloosa, AL 35487, U.S.A. \\ $P$. van der Laan \\ Department of Mathematics and Computing Science \\ Eindhoven University of Technology \\ Eindhoven, The Netherlands \\ M. A. van de Wiel \\ Department of Mathematics and Computing Science \\ Eindhoven University of Technology \\ Eindhoven, The Netherlands
}

\begin{abstract}
Distribution-free Shewhart-type control charts are proposed for future sample percentiles based on a reference sample. These charts have a key advantage that their in-control run length distribution do not depend on the underlying continuous process distribution. Tables are given to help implement the charts for given sample sizes and false alarm rates. Expressions for the exact run length distribution and the average run length $(A R L)$ are obtained using expectation by conditioning. Properties of the charts are studied, via evaluations of the run length distribution and the $A R L$. These computations show that in certain cases the proposed charts have attractive $A R L$ properties over standard parametric charts such as the CUSUM and the EWMA. Calculations are illustrated with several short examples. Also included is a numerical example, using data from Montgomery (1997), where an application of the precedence chart produced slightly different results.
\end{abstract}

Key Words and Phrases: Statistical Process Control; Nonparametric; Order Statistics; Quantiles; Precedence Statistics; False Alarm Rate; Average Run Length; Lehmann Alternatives; Proportional Hazards Alternatives.

\footnotetext{
${ }^{1}$ This is the companion report; edited and revised version submitted for publication; June 2000.
} 


\section{Introduction}

It is well known that nonparametric (hereafter NP) or distribution-free control charts can be useful in a variety of statistical process control (SPC) problems. Although these charts have not been the typical choice in traditional SPC applications, recently there is renewed interest in such methods and several authors have discussed where and why such procedures can be appealing; see for example, the recent papers by Woodall (2000) and Woodall and Montgomery (1999). Intuitively, it is expected that control charts designed with a particular parametric distribution (such as the normal) in mind will be "less efficient" when the true distribution is different and the loss of efficiency will depend on how far the departure actually is. For example, according to Hawkins and Olwell (1998; p. 76), "CUSUM (charts) designed for normal processes can have a much higher false alarm rate for skewed or heavy-tailed data". With such a motivation, Chakraborti, Van der Laan and Bakir (2000) (hereafter CVB) recently surveyed the literature on univariate variables charts that are nonparametric (NP) or distribution-free in the sense that the in-control run length distrution of the charts do not depend on the underlying process distribution. Besides being quite easy to implement, one key advantage of the distribution-free charts is that one does not need to assume any particular distribution (such as the normal) for the underlying process and the in-control probability calculations and associated conlusions remain valid for any continuous distribution. Also, since nonparametric tests can be more efficient than their parametric counterparts when sampling from skewed or heavy-tailed distributions, one would expect the NP charts to be "better" than the parametric charts in certain situations.

One of the NP charts reviewed by CVB is a two-sided Shewhart-type chart proposed by Janacek and Meikle (1997) (hereafter JM), which tracks the medians of independent test samples, with limits based on an in-control (or, a) reference sample. These authors noted some practical advantages of using median-based control charts. Broadly speaking, these charts are useful since medians are far 
less sensitive to measurement errors and medians can be determined (from ranks, for example) in situations where measures of quality involve, say, a "subjective assessment of ordinal data". In this paper the JM charts are examined in more detail, various properties are studied and generalizations are proposed. First, it is shown that their procedure is based on the "control median statistic" of Mathisen (1943). With this connection, in general, a class of distribution-free two-sample test statistics known as "precedence" statistics is employed to construct a chart for a test (future) sample quantile, including the median. Thus, for example, one can constructa chart for the $75^{\text {th }}$ percentile (0.75-quantile) of a future test sample. The event when a chart signals (sounds an alarm) an out-of-control situation is called a signaling event and the special signaling event when the process is actually in-control is called a false alarm. Clearly, one would like the probability of a false alarm, or the false alarm rate, to be very small. The in-control distribution of the precedence statistic is given, which is the same for all underlying continuous distributions (distribution-free), so that the false alarm probability is the same for all continuous distributions. For the proposed charts, the exact probability of a false alarm is explicitly derived in terms of the distribution of the precedence statistic. For a desired false alarm rate, the chart constants are obtained so that the chart can be implemented; a table is provided to aid in this process.

The performance of a control chart is usually judged in terms of the "run length" distribution. The run length random variable $N$ is discrete, taking values $1,2, \ldots$, which represents the number of subgroups (test samples) that are to be observed before a chart gives the first signal. The mean of the run length distribution, the so-called average run length $(A R L)$, is a popular summary measure of a chart's performance. It should be noted that even though we are dealing with a Shewhart-type chart, the run length distribution is not geometric since the signaling events are mutually dependent. As a result, the $A R L$ is not equal to the reciprocal of the probability of a signal. An expression for the exact run length distribution for the proposed chart is derived using a conditioning argument (see for 
example, Chakraborti, 2000) which yields the exact in-control run length distribution. Exact expressions for the $A R L$ and the exact in-control $A R L$ are found from this. A computer program has been developed to calculate the exact $A R L$ and some results are presented in Section 4. Robustness of the charts is examined, both for the in-control and the out-of-control situations, with respect to the $A R L$ relative to some parametric control charts. We also study the performance of the proposed chart by calculating the probability of detecting an out-of-control situation within $k$ samples for several values of $k$. For the special case $k=1$, these probabilities correspond to the operating characteristic $(O C)$ function (see for example, Montgomery, 1997; pp. 206-208) for which we derive exact expressions. We tabulated these probabilities for some well known parametric (such as the normal shift) and semi-parametric family of alternatives, namely the "Lehmann alternatives" and the "proportional hazards" alternatives. The latter two classes of distributions, used more frequently in the reliability literature, seem to offer a convenient compromise (and therefore some flexibility to the quality practitioner), between a completely parametric and a fully nonparametric formulation and solution. Some discussions on this can be found in Section 4. The effect of the size of the reference sample on the run length distribution and the false alarm rate is studied in Section 5. In Section 6 some results for the OC function are given. Finally, an example is given in Section 7 using data from Montgomery (2000) where an application of the precedence chart produced slightly different results. The proofs of some theorems are presented in the Appendix.

\section{Background and preliminaries}

First consider two-sided control charts, one-sided charts will be discussed later. Suppose that a random sample (hereafter referred to as the reference sample) of $m$ observations, $X_{1}, X_{2}, \ldots, X_{m}$, is available from an in-control process with an unknown continuous cumulative distribution function

(cdf) $F$. These observations are arranged in an ascending order and two order statistics, $X_{(a: m)}$ and 
$X_{(b: m)}$ (for given $1 \leq a<b \leq m$ ), are found. The two-sided control chart for a desired future sample quantile (i.e. an order statistic in the future sample) is given by the lower control limit $L C L=X_{(a: m)}$ and the upper control limit $U C L=X_{(b: m)}$. Now suppose in Phase II, test samples are drawn independently of one another and also of the reference sample and one is interested in checking whether or not the process is in-control. Let $Y_{\mathrm{h} 1}, Y_{\mathrm{h} 2}, \ldots, Y_{\mathrm{h}_{\mathrm{h}}}, h=1,2, \ldots$, denote the $h^{\text {th }}$ test sample of size $n_{h}$, and let $Y_{\left(j: n_{h}, h\right)}$ be the (some desired) $j^{\text {th }}$ order statistic in the $h^{\text {th }}$ test sample. "A process is incontrol" means that the reference distribution is the same as that of the test distribution. More precisely, if $G_{h}$ denotes the cdf of the distribution of the $h^{\text {th }}$ test sample, a process in-control at stage $h$ means that there is no significant evidence against $F=G_{h}$. For now, assume that the test samples are of the same size $n$ so that the subscript $h$ can be suppressed. After a test sample is collected, $Y_{(j: n)}$ is found and is compared with the control limits. If $Y_{(j: n)}$ lies between the control limits the process is declared to be in-control. On the other hand if $Y_{(j: n)}$ lies outside the control limits, the process is declared to be out-of-control and a search for assignable causes might be started.

\section{Charts based on precedence statistics}

Let $W_{j}$ denote the number of $X$-observations that "precede" (i.e. are not greater than) $Y_{(j: n)}$. In the literature, the statistic $W_{j}$ has been called a precedence statistic and a test based on $W_{j}$ has been called a "precedence test." Although equivalent forms of this test have been considered by other authors, the name "precedence test" seems to have been coined by Nelson $(1963,1993)$, who proposed it as a quick and simple nonparametric test for life-test data. Chakraborti and Van der Laan (hereafter CV) $(1996,1997)$ gave overviews of a rather substantial literature on precedence and precedence-type tests and confidence intervals for various problems including situations where the 
data contain right-censored observations. In this paper we consider NP or distribution-free control charts based on the precedence statistic. First we define a (NP, or) distribution-free control chart.

Definition: A control chart is distribution-free if its in-control run length distribution is the same for all continuous process distributions.

When the process is in-control (indicated by the subscript $C$ ), the exact probability distribution of the precedence statistic can be obtained (see for example, CV, 1996) either by mathematicalstatistical techniques or simply by combinatorial arguments. This is given by

$$
P_{C}\left(W_{j}=w\right)=\frac{\left(\begin{array}{c}
j+w-1 \\
w
\end{array}\right)\left(\begin{array}{c}
m+n-j-w \\
m-w
\end{array}\right)}{\left(\begin{array}{c}
m+n \\
m
\end{array}\right)}, w=0,1, \ldots, m .
$$

The following figures illustrate the distribution of $W_{j}$ for some selected values of $m, n$ and $j$. Note that when $n$ is odd and the chosen test sample quantile is the median, the distribution of $W_{j}$ is symmetric.
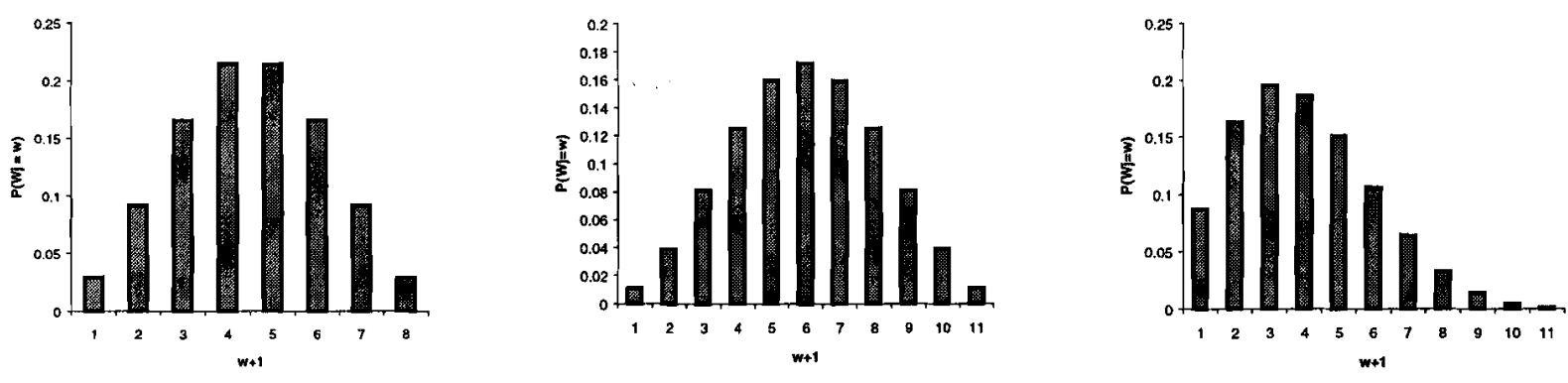

Figure 1:(a) $m=7, n=9$, $j=5$

(b) $m=10, n=9$, $j=5$

(c) $m=10, n=9$,

Note: These figures were done in Excel; it is necessary to show the horizontal scale from 1 to $(m+1)$

The important point is that the in-control distribution of $W_{j}$ depends only on $m, n$ and $j$ and not on the underlying process distributions $F$ or $G$. Thus, any decision rule based on $W_{j}$ will be distribution-free as long as the underlying distributions are continuous and identical. It therefore 
follows (and later explicitly shown) that the in-control run length distribution of these charts is distribution-free and thus, control charts based on precedence statistics are distribution-free. Note that if the $h^{\text {th }}$ test sample is of size $n_{h}$, the distribution of $W_{j}$ can be easily found from (1) by replacing $n$ by $n_{h}$. Thus a charting procedure based on a precedence statistic is flexible enough to accommodate variable test sample sizes.

The probability that the proposed chart "does not signal" can be expressed in terms of the precedence statistic $W_{j}$. This is true since the events that $Y_{(j: n)}$ lies between two $X$-order statistics $X_{(a: m)}$ and $X_{(b: m)}$ (where $\left.1 \leq a<b \leq m\right)$ and that the precedence statistic $W_{j}$ lies between $a$ and $b-1$, with both endpoints included, are the same. Thus,

$$
p=P\left(X_{(a: m)} \leq Y_{(j ; n)} \leq X_{(b: m)}\right)=P\left(a \leq W_{j} \leq b-1\right)=p\left(m, n, j ; F, G_{h}\right),
$$

say. Note that the coverage probability $p$ in (2) is in fact the $O C$ function of the proposed chart and that $1-$ the coverage probability $=$ the probability of an alarm. A general expression for $p$ is given in Section 4.

Let $p_{0}$ be the in-control value of $p$, so that $1-p_{0}$ is the probability of a false alarm or the false alarm rate. Thus, $p_{0}=P_{C}\left(a \leq W_{j} \leq b-1\right)=p(m, n, j ; F, F)$. Using (1) and (2), the chart constants $a$ and $b(1 \leq a<b \leq m)$ can be found such that

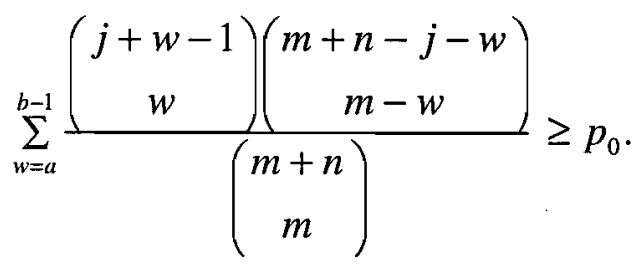

Note that the inequality in (3) is used to accommodate the discreteness of $W_{j}$. The control limits are typically found by setting $p_{0}$ (or $1-p_{0}$ ) to some desirable high (or low) value, say $P_{0}$ (or $1-P_{0}$ ), finding $a$ and $b$, and then locating $L C L=X_{(a: m)}$ and $U C L=X_{(b: m)}$.

Remark 1: In practice, the false alarm rate of a control chart is often specified to be equal to some given value (such as 0.0027). However, since the precedence statistic is a discrete random variable, 
there is a possibility that the specified false alarm probability will not be exactly achieved. In such situations, a conservative strategy (as given in (3)) is recommended, where the constants are determined such that $p_{0}$ is not smaller than the specified value (that is $p_{0} \geq P_{0}$ ), or in other words, the false alarm rate is not larger than $1-P_{0}$. Also, due to the discreteness of the statistic, note that not all desired $P_{0}$ values might be available for all combinations of $m, n$ and $j$, especially when $m$ and/or $n$ are small.

Remark 2: Sometimes it is desirable to design a chart with a specified in-control $A R L$ (say $A R L_{0}$ ). In our case, since the control limits are estimated, the signaling events are dependent (even though the test samples are independent) and so the run length distribution is not geometric. As a result, the relationship between $A R L_{0}$ and the false alarm rate is not a simple one. Using a conditioning argument, an exact expression for the in-control $A R L$ is derived in Section 4. In principle, this expression can be evaluated and solved for chart constants $a$ and $b$, for given values of $A R L_{0}, m, n$ and $j$. This, in principle, is not so hard. One can adopt a Newton-type algorithm that stops when the achieved $A R L$ is within a specified distance from the specified value. One needs to note that the $A R L$ is increasing in $b$ and decreasing in $a$. For the median, one can find $a$ and take $b=m-a+1$, which leads to a unique solution. For other quantiles more than one solutions are possible. Because of the complexity, however, we suggest finding $a$ and $b$ for a given false alarm rate. A computer program has been written for this purpose.

A second option might be to use a reference sample large enough (large $m$ ) such that the effects of estimating the limits (variation in the $X$-order statistics) can be virtually ignored. In that case $A R L_{0}=$ $\left(1-p_{0}\right)^{-1}$, so that for a specified $A R L_{0}$, one can take $\left(A R L_{0}-1\right) / A R L_{0}=p_{0}$ in (3) and solve for $a$ and $b$. The question of how large $m$ has to be for this to be appropriate will be addressed later.

\subsection{The median chart}

For purposes of illustration, we concentrate on the median chart. As noted in the introduction, the case of the median is important for at least two practical reasons. First, the median is a robust estimator of the location and is preferred in situations where "large" measurement errors are expected. Secondly, the median is a more flexible estimator of location applicable in more diverse situations unlike the mean. For example, the median can be determined only from the ranks of the measurements. Our basic approach, however, is general and can be used for other sample quantiles or percentiles.

For simplicity, let $n$ be odd, say $2 s+1$, so that the median of the $Y$-sample, $Y_{(j: n)}$, is uniquely defined with $j=s+1$. In this case, the precedence statistic $W_{s+1}$ is in fact the median statistic of Mathisen (1943). The in-control distribution of $W_{s+1}$, given by (1), is symmetric, and a reasonable 
choice for $b$ is $m-a+1$. Thus, using (3), the constant $a(\geq 1)$ can be determined as the largest integer such that

$$
\sum_{w=a}^{m-a} \frac{\left(\begin{array}{c}
s+w \\
w
\end{array}\right)\left(\begin{array}{c}
m+s-w \\
m-w
\end{array}\right)}{\left(\begin{array}{c}
m+2 s+1 \\
m
\end{array}\right)} \geq P_{0},
$$

where $1-P_{0}$ is the specified value of the false alarm rate. After $a$ is found, $b$ is taken to be $m-a+1$.

Note that using symmetry, (4) can be written as $P_{C}\left(0 \leq W_{j} \leq a-1\right) \leq\left(1-P_{0}\right) / 2$ and this is more convenient to work with in practice. A computer program was written to solve equation (4). In Table $1,(a, b=m-a+1)$ values are provided for $m=50,100,500,1000, n=5,11,25$ and $P_{0}=0.99,0.995$, 0.9973. In each case, the values of $P_{C}\left(0 \leq W_{j} \leq a-1\right)$ and $P_{C}\left(m-a+1 \leq W_{j} \leq m\right)$ are also given (together with the actual value of the in-control average run length; to be discussed later). To calculate the actual exact false alarm rate, one simply adds these to probabilities. Outside the range of the table one can use a direct enumeration or a computer program available from the authors. Note also that the process of finding $a$ (hence the control chart) is similar (but not the same) to that of finding a confidence interval for the median of a continuous population based on the sign statistic (see for example, Gibbons and Chakraborti, 1992).

Example: Suppose $m=50$ and $n=5$. Then $j=3$, corresponding to the test sample median. Let $P_{0}$ to be 0.99 so that the nominal false alarm rate is 0.01 . From Table 1 , we get $a=3$ and $b=48$ with $P_{C}\left(X_{(3: 50)} \leq Y_{(3: 5)} \leq X_{(48: 50)}\right)=1-2 * 0.0036=0.9928$. Thus, the distribution-free control chart is given by the $3^{\text {rd }}$ and the $48^{\text {th }}$ reference sample order statistics, i.e., $L C L=X_{(3: 50)}$ and $U C L=X_{(48: 50)}$. The exact false alarm rate achieved for this chart is equal to $2 * 0.0036=0.0072$.

Remark 3: It may be noted that the achieved false alarm rate of 0.0072 in the example is smaller than the nominal value of 0.01 . This is consistent with remark 1 , given the discrete in-control distribution of $W_{j}$, the given values of $m, n, j$, and the desired nominal false alarm rate $1-P_{0}$. Also, as was indicated earlier, this is a common occurrence when dealing with many nonparametric procedures whether used for usual hypothesis testing or for setting up confidence intervals. For larger values of $m$, however, this is less likely to be the situation. For example, if $m=500$ instead, the same tables yield $a=40$ so that $L C L=X_{(40: 500)}$ and $U C L=X_{(461: 500)}$, with the actual false alarm rate equal to $2 * 0.00477=0.00954$, much closer to the nominal value. 
Remark 4: An important practical question for the NP chart user is: what false alarm rates are available for choosing, given the values of $m, n$ and $j$. In practice, $m$ will probably be larger than $n$ since it is often easier to obtain historical or reference data. For $m=50,100,500,1000, n=5,11,25$ and $j=(n+1) / 2$, the achievable false alarm probabilities can be seen in Table 1 .

Table 1. Precedence charts for test sample median and in-control ARL values

\begin{tabular}{|c|c|c|c|c|c|c|}
\hline$\overline{P_{0}}$ & $n$ & JNm & 50 & 100 & 500 & 1000 \\
\hline \multirow{3}{*}{0.9900} & 5 & 3 & $\begin{array}{c}(3,48) \\
(0.0036,0.0036) \\
635.7\end{array}$ & $\begin{array}{c}(7,94) \\
(0.00432,0.00432) \\
214.9\end{array}$ & $\begin{array}{c}(40,461) \\
(0.00477,0.00477) \\
114.5\end{array}$ & $\begin{array}{c}(82,919) \\
(0.00499,0.00499) \\
104.6\end{array}$ \\
\hline & 11 & 6 & $\begin{array}{c}(7,44) \\
(0.00465,0.00465) \\
642.2\end{array}$ & $\begin{array}{c}(15,86) \\
(0.00428,0.00428) \\
\ldots \quad 245.0\end{array}$ & $\begin{array}{c}(83,418) \\
(0.00494,0.00494) \\
113.3\end{array}$ & $\begin{array}{c}(167,834) \\
(0.00487,0.00487) \\
108.4\end{array}$ \\
\hline & 25 & 13 & $\begin{array}{c}(10,41) \\
(0.00305,0.00305) \\
10990.0\end{array}$ & $\begin{array}{c}(23,78) \\
(0.00402,0.00402) \\
510.8\end{array}$ & $\begin{array}{c}(127,374) \\
(0.00473,0.00473) 1 \\
128.3\end{array}$ & $\begin{array}{c}(258,743) \\
(0.00497,0.00497) \\
109.8\end{array}$ \\
\hline \multirow{3}{*}{0.9950} & 5 & 3 & $\begin{array}{c}(2,49) \\
(0.0015,0.0015) \\
5671.0\end{array}$ & $\begin{array}{l}(5,96) \\
(0.00176,0.00176) \\
678.4\end{array}$ & $\begin{array}{c}(31,470) \\
(0.00233,0.00233) \\
242.3\end{array}$ & $\begin{array}{c}(64,937) \\
(0.00246,0.00246) \\
215.1\end{array}$ \\
\hline & 11 & 6 & $\begin{array}{c}(5,46) \\
(0.00125,0.00125) \\
9503.0\end{array}$ & $\begin{array}{c}(13,88) \\
(0.00225,0.00225) \\
574.5\end{array}$ & $\begin{array}{c}(72,429) \\
(0.0024,0.0024) \\
240.9\end{array}$ & $\begin{array}{c}(146,855) \\
(0.00244,0.00244) \\
219.8\end{array}$ \\
\hline & 25 & 13 & $\begin{array}{c}(9,42) \\
(0.00155,0.00155) \\
44750.0\end{array}$ & $\begin{array}{c}(21,80) \\
(0.002,0.002) \\
1488.0\end{array}$ & $\begin{array}{c}(118,383) \\
(0.00243,0.00243) \\
261.0\end{array}$ & $\begin{array}{c}(239,762) \\
(0.00245,0.00245) \\
227.5\end{array}$ \\
\hline \multirow{3}{*}{0.9973} & 5 & 3 & $\begin{array}{c}(1,50) \\
(0.0004,0.0004) \\
\infty\end{array}$ & $\begin{array}{c}(4,97) \\
(0.00102,0.00102) \\
1550.0\end{array}$ & $\begin{array}{c}(25,476) \\
(0.00127,0.00127) \\
460.2\end{array}$ & $\begin{array}{c}(51,950) \\
(0.00129,0.00129) \\
419.5\end{array}$ \\
\hline & 11 & 6 & $\begin{array}{c}(5,46) \\
(0.00125,0.00125) \\
9503.0\end{array}$ & $\begin{array}{c}(11,90) \\
(0.00106,0.00106) \\
1630.0\end{array}$ & $\begin{array}{c}(64,437) \\
(0.00131,0.00131) \\
456.1\end{array}$ & $\begin{array}{c}(130,871) \\
(0.00131,0.00131) \\
409.8\end{array}$ \\
\hline & 25 & 13 & $\begin{array}{c}(8,43) \\
(0.00075,0.00075) \\
173700.0\end{array}$ & $\begin{array}{c}(19,82) \\
(0.00092,0.00092) \\
5183.0\end{array}$ & $\begin{array}{c}(110,391) \\
(0.00127,0.00127) \\
526.2\end{array}$ & $\begin{array}{c}(224,777) \\
(0.00133,0.00133) \\
430.2\end{array}$ \\
\hline
\end{tabular}


For completeness and later reference, the steps of the proposed control charting procedure are enumerated below.

Step 1 Start with a reference sample of size $m$.

Step 2 Select a test sample size $n$. Select the test sample quantile (for example, the median) to be monitored. This determines $j$ for a given $n$.

Step 3 Specify the false alarm rate $1-P_{0}$. Find, using (4), the constant $a$. Use Table 1 (or evaluate using (4) directly) for this purpose. Control limits for the median chart are: $L C L=X_{(a: m)}$ and $U C L=$ $X_{(m-a+1: m)}$.

Step 4 Begin collecting test samples, each of size $n$. Find the value of the $j^{\text {th }}$ order statistic for each test sample. If any of the values falls outside the control limits, the process might not be in the desired state of control.

\subsection{Control charts for other sample quantiles}

Situations might arise in practice where quantiles other than the median of the test samples are of interest. For example, one might be interested in the $20^{\text {th }}$ or the $75^{\text {th }}$ percentile of the distribution. Here, in principle, one could proceed as outlined in steps $1-4$, but since the distribution of $W_{j}$ is not symmetric in this case, the choice of $a$ and $b$ is more involved. We propose the following "equaltailed" procedure, when the $100 q^{\text {th }}(0<q<1)$ percentile is of interest.

Find the largest integer $a(1 \leq a \leq[m q])$ such that

$$
P_{C}\left(0 \leq W_{j} \leq a-1\right) \leq \frac{\left(1-P_{0}\right)}{2}
$$

and the smallest integer $b(a<b \leq m)$ such that

$$
P_{C}\left(b \leq W_{j} \leq m\right) \leq \frac{\left(1-P_{0}\right)}{2} .
$$

Note that this procedure is similar to setting up the critical region for, say the sign test, for an arbitrary population quantile. 
A computer program has been developed to solve these equations; results for some combinations of $m, n, j$ and $P_{0}$ are given in Table 2 .

Table 2. Precedence charts for other test sample quantiles and in-control ARL values

\begin{tabular}{|c|c|c|c|c|c|c|}
\hline$P_{0}$ & $\bar{n}$ & $j \mathrm{Jm}$ & 50 & 100 & 500 & 1000 \\
\hline \multirow{9}{*}{0.9900} & 10 & 3 & $(1,35)$ & $(2,68)$ & $(17,327)$ & $(36,651)$ \\
\hline & & & $(0.00351,0.00475)$ & $(0.00212,0.00439)$ & $(0.00447,0.00493)$ & $(0.00492,0.00498)$ \\
\hline & & & 890.8 & 366.5 & 121.7 & 108.0 \\
\hline & 15 & 6 & $(4,38)$ & $(10,72)$ & $(56,348)$ & $(115,692)$ \\
\hline & & & $(0.00333,0.00346)$ & $(0.00452,0.00467)$ & $(0.00461,0.00477)$ & $(0.00491,0.00489)$ \\
\hline & & & 2144.0 & 271.8 & 123.0 & 109.1 \\
\hline & 20 & 15 & $(19,49)$ & $(41,94)$ & $(217,461)$ & $(437,919)$ \\
\hline & & & $(0.00333,0.00168)$ & $(0.00413,0.00499)$ & $(0.0048,0.00461)$ & $(0.00489,0.00473)$ \\
\hline & & & 13800.0 & 378.6 & 127.5 & 113.4 \\
\hline \multirow{9}{*}{0.9950} & 10 & 3 & No & $(2,71)$ & $(13,344)$ & $(28,684)$ \\
\hline & & & Solution & $(0.00211,0.00236)$ & $(0.00219,0.0024)$ & $(0.00247,0.00247)$ \\
\hline & & & & 648.1 & 260.8 & 221.1 \\
\hline & 15 & 6 & $(3,39)$ & $(8,75)$ & $\overline{(49,360)}$ & $(100,717)$ \\
\hline & & & $(0.00128,0.0021)$ & $(0.00181,0.00215)$ & $(0.00241,0.00249)$ & $(0.00245,0.00248)$ \\
\hline & & & 10300.0 & 870.4 & 244.0 & 220.8 \\
\hline & 20 & 15 & $(18,49)$ & $(39,96)$ & $(204,466)$ & $(412,930)$ \\
\hline & & & $(0.00206,0.00168)$ & $(0.00249,0.00142)$ & $(0.00238,0.00246)$ & $(0.00248,0.00235)$ \\
\hline & & & 31030.0 & 1282.0 & 260.6 & 231.0 \\
\hline \multirow{9}{*}{0.9973} & 10 & 3 & No & $(1,74)$ & $(10,357)$ & $(22,710)$ \\
\hline & & & Solution & $(0.00056,0.00119)$ & $(0.00109,0.0013)$ & $(0.00127,0.00133)$ \\
\hline & & & & 2309.0 & 523.0 & 428.3 \\
\hline & 15 & 6 & $(3,40)$ & $(7,77)$ & $(43,371)$ & $(88,738)$ \\
\hline & & & $(0.00128,0.00122)$ & $(0.00105,0.00121)$ & $(0.00126,0.00129)$ & $(0.00128,0.00132)$ \\
\hline & & & 22550.0 & 1964.0 & 487.8 & 426.6 \\
\hline & & 15 & $(17,50)$ & $(36,97)$ & $(194,471)$ & $(391,938)$ \\
\hline & & & $(0.00124,0.0003)$ & $(0.00110,0.00064)$ & $(0.00133,0.00118)$ & $(0.00134,0.0013)$ \\
\hline & & & 485900.0 & 4599.0 & 527.5 & 429.6 \\
\hline
\end{tabular}


Example: Suppose $m=100, n=20$ and that the $75^{\text {th }}$ percentile of the future sample is of interest. From Table 2, for $j=15$ and a nominal false alarm rate of $0.01\left(P_{0}=0.99\right)$, we find $L C L=X_{(41: 100)}$ and $U C L=X_{(94: 100)}$ which give a control chart with an exact false alarm rate of $0.00413+0.00499=$ 0.00912 . On the other hand, for a nominal false alarm rate of $0.0027\left(P_{0}=0.9973\right)$, we get $L C L=$ $X_{(36: 100)}$ and $U C L=X_{(97: 100)}$, with an exact false alarm rate of 0.00174 .

\subsection{One-sided charts}

The required modifications are simple for the one-sided charts. If detecting higher values (whether the parameter of interest has shifted to the right) is of interest one can use $U C L=X_{(b: m)}$, where the $b$ is found from $P_{C}\left(Y_{(j: n)} \geq X_{(b: m)}\right) \leq 1-P_{0}$. However, this condition is the same as $P_{C}\left(b \leq W_{j} \leq m\right) \leq 1-P_{0}$, which is basically (6), except that the right hand side is doubled. Thus, for a nominal false alarm rate of $0.0027, b$ can be simply found by doubling the tail probability requirement from 0.00135 to 0.0027 and using (the same program used for) Table 2 . In general, one can use the program for the two-sided chart setting $P_{0}^{(2)}=1-2\left(1-P_{0}^{(1)}\right)$, and use the resulting upper control limit as the one-sided $U C L$.

Example: Let $m=75, n=15, j=8$ and let the nominal false alarm rate be 0.0027 . Thus, 1 $P_{0}^{(1)}=0.0027$. Using the same computer program developed for Table 1 , with $P_{0}^{(2)}=1-2 * 0.0027=$ 0.9946 , we get $P_{C}\left(Y_{(8: 15)} \geq X_{(64: 75)}\right)=0.00251$ so that $b=64$ and the one-sided chart is given by $U C L$ $=X_{(64: 75)}$ with an exact false alarm rate of 0.00251 .

Similarly, in detecting lower values (whether parameter is shifted to the left) one can use $L C L$ $=X_{(a: m)}$, where $a$ can be found from (5) by setting the right hand side equal to $\left(1-P_{0}\right)$.

The performance of a control chart is often studied with respect to its run length distribution, particularly in Phase II applications. The run length random variable is $N$, the number of test samples to be collected before the chart gives the first signal of a change. This is discussed in the following. 


\section{Run length distribution}

For ease of presentation, first consider the upper one-sided chart given by $U C L=X_{(b: m)}$. The run length distribution can be obtained as follows. Given $X_{(b: m)}$, the run length random variable $N$ has a geometric distribution with probability, say, $p_{u}=P\left(Y_{(j: n)} \leq X_{(b: m)}\right)$. Thus, the unconditional distribution of $N$ is given by

$$
P(N=k)=E_{X_{(b m)}}\left(p_{u}^{k-1}-p_{u}^{k}\right) \text { for } k=1,2, \ldots
$$

The quantity $E_{X_{(b, m)}}\left(p_{u}^{k}\right)$ can be calculated by noting that

$$
p_{u}=P\left(Y_{(j ; n)} \leq X_{(b: m)}\right)=P\left(U_{(j ; n)} \leq G F^{-1}\left\{U_{(b ; m)}\right\}\right)
$$

where $U_{(e: l)}$ is the $e^{\text {th }}$ order statistic of a sample of size $l$ from the Uniform $(0,1)$ distribution. Thus,

$$
\begin{aligned}
E_{X_{t b m, m}} & \left(p_{u}^{k}\right) \\
= & E_{U_{(b ; m)}}\left[P\left(U_{(j \cdot n)} \leq G F^{-1}\left\{U_{(b ; m)}\right\}\right)\right]^{k} \\
& =\int_{0}^{1}\left[P\left(U_{(j ; n)} \leq G F^{-1}(t)\right)\right]^{k} f(t) d t \\
& =\int_{0}^{1}\left[\int_{0}^{G F^{-1}(t)} \frac{1}{B(j, n-j+1)} u^{j-1}(1-u)^{n-j} d u\right]^{k} f(t) d t,
\end{aligned}
$$

where $f(t)$ is the density function of $U_{(b \cdot m)}$. Simplifying this expression further, we get

$$
\begin{aligned}
E_{X_{(b m)}}\left(p_{u}^{k}\right) & =\int_{0}^{1}\left[\frac{1}{B(j, n-j+1)} \sum_{h=0}^{n-j} \frac{(-1)^{h}}{j+h}\left(\begin{array}{c}
n-j \\
h
\end{array}\right)\left\{G F^{-1}(t)\right\}^{j+h}\right]^{k} \frac{m !}{(b-1) !(m-b) !} t^{h-1}(1-t)^{m-b} d t \\
& =D_{u}^{*}(k),
\end{aligned}
$$

say. The run length probability distribution for the upper one-sided chart is given by

$$
P(N=k)=D_{u}^{*}(k-1)-D_{u}^{*}(k), k=1,2, \ldots, \text { and } D_{u}^{*}(0)=1 .
$$

For the two-sided charts one can proceed along the same lines. Here, given the control limits, $N$ follows a geometric distribution with probability $p$ given in (2). Thus for $k=1,2, \ldots$. 


$$
P(N=k)=E_{X_{(a: m)}, X_{(b: m)}}\left(p^{k-1}-p^{k}\right)
$$

The quantity $E_{X_{(a: m)}, X_{(b: m)}}\left(p^{k}\right)$ can be calculated by noting that

$$
\begin{gathered}
p=P\left(X_{(a: m)} \leq Y_{(j: n)} \leq X_{(b: m)}\right) \\
=P\left(G F^{-1}\left\{U_{(a: m)}\right\} \leq U_{(j: n)} \leq G F^{-1}\left\{U_{(b: m)}\right\}\right),
\end{gathered}
$$

so that

$$
\begin{aligned}
& E_{X_{(a: m)}, X_{(b: m)}}\left(p^{k}\right) \\
& =E_{U_{(a: m)}, U_{(b ; m)}}\left[P\left\{G F^{-1}\left(U_{(a: m)}\right) \leq U_{(j: n)} \leq G F^{-1}\left(U_{(b: m)}\right)\right\}\right]^{k} \\
& =\int_{0}^{1} \int_{0}^{t}\left[P\left\{G F^{-1}(s) \leq U_{(j: n)} \leq G F^{-1}(t)\right\}\right]^{k} f(s, t) d s d t \\
& =\int_{0}^{1} \int_{0}^{t}\left[\int_{G F^{-1}(s)}^{G F^{-1}(t)} \frac{1}{B(j, n-j+1)} u^{j-1}(1-u)^{n-j} d u\right]^{k} f(s, t) d s d t,
\end{aligned}
$$

where $f(s, t)$ is the joint density function of $U_{(a: m)}$ and $U_{(b: m)}$ of a random sample of size $m$ from the Uniform( 0,1$)$ distribution. Substituting the formula for this joint density we get

$$
\begin{aligned}
E_{X_{(a-m)}, X_{(b-m)}}\left(p^{k}\right)=\int_{00}^{1} \int_{00}^{t}\left[\frac{1}{B(j, n-j+1)} \sum_{h=0}^{n-j} \frac{(-1)^{h}}{j+h}\left(\begin{array}{c}
n-j \\
h
\end{array}\right)\left\{\left(G F^{-1}(t)\right)^{j+h}-\left(G F^{-1}(s)\right)^{j+h}\right\}\right]^{k} \\
\\
\quad \frac{m !}{(a-1) !(b-a-1) !(m-b) !} s^{a-1}(t-s)^{b-a-1}(1-t)^{m-b} d s d t \\
=D^{*}(k),
\end{aligned}
$$

say. The run length probability distribution for the two-sided chart is given by

$$
P(N=k)=D^{*}(k-1)-D^{*}(k), \quad k=1,2, \ldots, \text { and } \quad D^{*}(0)=1 .
$$

Now we look at some special situations. First consider the in-control case.

\subsection{In-control run length distribution}

For the one-sided chart, using (8), the in-control run length distribution is given by 


$$
P_{C}(N=k)=D_{u}(k-1)-D_{u}(k), \quad k=1,2, \ldots, D_{u}(0)=1,
$$

and

$$
D_{u}(k)=\int_{0}^{1}\left[\frac{1}{B(j, n-j+1)} \sum_{h=0}^{n-j} \frac{(-1)^{h}}{j+h}\left(\begin{array}{c}
n-j \\
h
\end{array}\right) t^{j+h}\right]^{k} \frac{m !}{(b-1) !(m-b) !} t^{a-1}(1-t)^{m-b} d t .
$$

For the two-sided chart, using (9), the in-control run length distribution is given by

$$
P_{C}(N=k)=D(k-1)-D(k), \quad k=1,2, \ldots, D(0)=1,
$$

and

$$
\begin{aligned}
D(k)=\int_{0}^{1} \int_{0}^{t}\left[\frac{1}{B(j, n-j+1)} \sum_{h=0}^{n-j} \frac{(-1)^{h}}{j+h}\left(\begin{array}{c}
n-j \\
h
\end{array}\right)\left(t^{j+h}-s^{j+h}\right)\right]^{k} \\
\frac{m !}{(a-1) !(b-a-1) !(m-b) !} s^{a-1}(t-s)^{b-a-1}(1-t)^{m-b} d s d t .
\end{aligned}
$$

Expressions (11) and (12) can be evaluated to find the entire in-control run length distribution or any specific in-control run length percentile of interest.

Remark 5: By definition $D(1)$ equals $P_{C}\left(X_{(a: m)} \leq Y_{(j: n)} \leq X_{(b: m)}\right)$, when the process is in-control. The expression for $D(1)$ can be evaluated directly, yielding

$$
D(1)=\frac{m !}{(a-1) !(b-a-1) !(m-b) !} \frac{\sum_{h=0}^{n-j} \frac{(-1)^{h}}{j+h}\left(\begin{array}{c}
n-j \\
h
\end{array}\right) B(j+b+h, m-b+1)[B(a, b-a)-B(j+h+a, b-a)]}{B(j, n-j+1)} .
$$

This gives an alternative expression for the probability $p_{0}$.

\subsection{Average run length (ARL)}

One popular characteristic of the run length distribution is the average or the mean of the run length distribution, the so-called $A R L$. From a practical standpoint, it is desirable that the in-control $A R L$ of the chart be "high", whereas the out-of-control $A R L$ be "low". The $A R L$ of the chart can be calculated from the run length distribution discussed above. 
Remark 6: An alternative view of the $A R L$ : Given the $X$-order statistics (the lower and the upper control limits), the run length distribution is geometric with probability $p$, where $p$ is given in (2), so that the conditional $A R L$ equals $\sum_{k=0}^{\infty} P(N>k)=\sum_{k=0}^{\infty} p^{k}$. The unconditional $A R L$ can be found by calculating the expectation of the conditional $A R L$ with respect to the joint distribution of the two Xorder statistics. Thus, for example, for the two-sided chart, the unconditional $A R L=$ $\sum_{k=0}^{\infty} E_{X_{(a: m)}, X_{(b: m)}} p^{k}=\sum_{k=0}^{\infty} D^{*}(k)$ and hence, for the in-control situation, $A R L_{0}=\sum_{k=0}^{\infty} D(k)$. These expressions clearly show that the in-control $A R L$ of the charts does not depend on either $F$ or $G$, in other words, the proposed chart is distribution-free.

It should be noted that for both the one-sided and the two-sided charts, certain conditions on the constants $a, b, j, m$ and $n$ need to be satisfied in order for the in-control $A R L$ to be finite. These are important practical consideration in order to implement the charts. The necessary conditions are given in the following theorem.

\section{Theorem 1}

(a) For the one-sided precedence control chart, $A R L_{0}<\infty$ iff $(m-b)-(n-j)>0$.

(b) For the two-sided precedence control chart, $A R L_{0}<\infty$ iff $(a-j)(n-j+1)+j(m-b+1)>0$.

Proof: The proof is given in the Appendix.

Remark 7: For the median chart take $b=m-a+1$ everywhere above.

Using Remark 6, the in-control and out-of-control $A R L$ of the proposed charts are obtained and shown below.

\subsubsection{In-control $A R L$}

Assuming that it exists, the in-control $A R L$, for the one-sided chart is given by

$$
A R L_{0}^{(1)}=\sum_{k=0}^{\infty} D_{u}(k)=\int_{0}^{1}\left[1-C^{*}(t, j, n)\right]^{-1} \frac{m !}{(b-1) !(m-b) !} t^{b-1}(1-t)^{m-b} d t
$$

where 


$$
C^{*}(t, j, n)=\frac{1}{B(j, n-j+1)} \sum_{h=0}^{n-j} \frac{(-1)^{h}}{j+h}\left(\begin{array}{c}
n-j \\
h
\end{array}\right){ }_{t}^{j+h}
$$

Assuming that it exists, the in-control $A R L$ for the two-sided chart is given by

$$
\begin{aligned}
A R L_{0}^{(2)}= & \sum_{k=0}^{\infty} D(k) \\
= & \int_{00}^{1 t} \int[1-C(s, t, j, n)]^{-1} \frac{m !}{(a-1) !(b-a-1) !(m-b) !} \\
& \quad s^{a-1}(t-s) b-a-1(1-t){ }^{m-b} d s d t,
\end{aligned}
$$

where

$$
C(s, t, j, n)=\frac{1}{B(j, n-j+1)} \sum_{h=0}^{n-j} \frac{(-1)^{h}}{j+h}\left(\begin{array}{c}
n-j \\
h
\end{array}\right)\left(t^{j+h}-s^{j+h}\right) .
$$

The $A R L_{0}$ values are evaluated for some two-sided charts with a given probability of false alarm such as 0.90 or 0.9973 . These charts and the corresponding $A R L_{0}$ values are shown in Tables 1 and 2 , for the median and other quantiles, respectively.

Now let us consider the out-of-control $A R L$.

\subsubsection{Out-of-control $A R L$}

For the one-sided chart, the $A R L$ is given by

$$
A R L^{(1)}=\int_{0}^{1}\left[1-F_{1}(t, j, n, F, G)\right]^{-1} \frac{m !}{(b-1) !(m-b) !} t^{b-1}(1-t)^{m-b} d t
$$

where

$$
F_{1}(t, j, n, F, G)=\frac{1}{B(j, n-j+1)} \sum_{h=0}^{n-j} \frac{(-1)^{h}}{j+h}\left(\begin{array}{c}
n-j \\
h
\end{array}\right)\left[G F^{-1}(t)\right]^{j+h}
$$

For the two-sided chart, the $A R L$ is given by

$$
A R L^{(2)}=\int_{00}^{1} \int_{0}^{t}\left[1-F_{2}(s, t, j, n, F, G)\right]^{-1} \frac{m !}{(a-1)(b-a) !(m-b) !} s^{a-1}(t-s)^{b-a-1}(1-t)^{m-b} d s d t,
$$


where

$$
F_{2}(s, t, j, n, F, G)=\frac{1}{B(j, n-j+1)} \sum_{h=0}^{n-j} \frac{(-1)^{h}}{j+h}\left(\begin{array}{c}
n-j \\
h
\end{array}\right)\left[\left\{G F^{-1}(t)\right\}^{j+h}-\left\{G F^{-1}(s)\right\}^{j+h}\right] .
$$

The out-of-control $A R L$ values are evaluated and commented upon later.

\subsection{Run length distribution and $A R L$ under some specific alternatives}

\subsubsection{Run length distribution}

As noted before, the run length distribution is of interest in studying the performance of a control chart. For example, one might ask, if the process mean has shifted from the in-control value, what is the probability that the chart will signal on the first test sample, or on the second test sample, or, in general, on the $k^{\text {th }}$ test sample? As also noted earlier, the distribution of $N$ is not geometric when the limits are estimated so that standard formulas based on the geometric distribution are not appropriate. General expressions for the run length distribution were given earlier ((8) and (10), respectively). It is evident that the run length distribution depends, in general, on the distrbution functions $F$ and $G$, through the composite function $\psi=G F^{-1}$. Depending on the assumptions made on $F$ and $G$, one can calculate the function $\psi$ and thus evaluate the run length distribution. For example, when $F=G, \psi(u)=u$ and the in-control run length distribution is obtained. Towards this end, the following theorem is useful. It may be noted that $\psi(u)=G F^{-1}(u), 0<u<1$, is in fact, a cdf its with support on the interval $(0,1)$. The following results have direct applications to what follows next. 


\section{Theorem 2}

(a) For location alternatives, $F(x)=H\left(x-\theta_{1}\right)$ and $G(x)=H\left(x-\theta_{2}\right)$, where $H$ is a continuous cdf, $x \in \Re$ and $\theta_{1}, \theta_{2} \in \Re, \psi(u)=H\left(\theta_{1}-\theta_{2}+H^{-1}(u)\right)$.

(b) For scale alternatives, $F(x)=H\left(x / \gamma_{1}\right)$ and $G(x)=H\left(x / \gamma_{2}\right)$, where $H$ is a continuous $\operatorname{cdf} x \in \Re$ and $\gamma_{1}, \gamma_{2} \in \Re^{+}, \psi(u)=H\left(\frac{\gamma_{1}}{\gamma_{2}} H^{-1}(u)\right)$.

(c) For location-scale alternatives, $F(x)=H\left(\frac{x-\theta_{1}}{\gamma_{1}}\right)$ and $G(x)=H\left(\frac{x-\theta_{2}}{\gamma_{2}}\right)$, where $H$ is a continuous cdf, $x \in \Re, \theta_{1}, \theta_{2} \in \Re$ and $\gamma_{1}, \gamma_{2} \in \Re^{+}$,

$$
\psi(u)=H\left(\frac{\theta_{1}-\theta_{2}}{\gamma_{2}}+\frac{\gamma_{1}}{\gamma_{2}} H^{-1}(u)\right)
$$

(d) For "Lehmann alternatives," $G(x)=F^{\delta}(x)$, where $x \in \Re$ and $\delta \in \Re^{+}, \psi(u)=u^{\delta}$.

(e) For "proportional-hazards" alternatives, $G(x)=1-[1-F(x)]^{\gamma}$, where $x \in \mathfrak{R}$ and $\gamma \in \mathfrak{R}^{+}, \psi(u)=1-(1-u)^{\gamma}$.

Remark 8: It is clear that the run length distribution can be calculated when the change from incontrol to out-of-control, or, more generally, the relationship between $F$ and $G$ is specified through $\psi$. If the underlying distributions are, for example, normal with variance 1 , Theorem 2, part (a), gives $\psi(u)=\Phi\left(\Phi^{-1}(u)-\theta\right)$, where $\theta=\theta_{2}-\theta_{1}$ so that the change is described in terms of the difference between the means of the distributions. The real problem, of course, is that in many practical situations the underlying distributions can not be completely specified. In the context of nonparametric (rank) tests, Lehmann (1953) suggested using (the model) $G=F^{\delta}$, where $\delta>0$, is an unknown parameter that describes how $F$ and $G$ differ. This relationship is known as the "Lehmann alternatives (LE)." Another interpretation is that when $\delta$ is a positive integer, $G$ is the cdf of the maximum of $\delta$ i.i.d. random variables with $\operatorname{cdf} F$. The assumed relationship between $F$ and $G$ is a "semi-parametric" formulation, with a practical advantage that under this model, power calculations of various rank tests are greatly simplified. Clearly, when $\delta=1$ the process is in-control. It appears that these alternatives have an important role to play in the context of (nonparametric) control charts. An example of a family of densities belonging to this class is shown in Figure 2. 


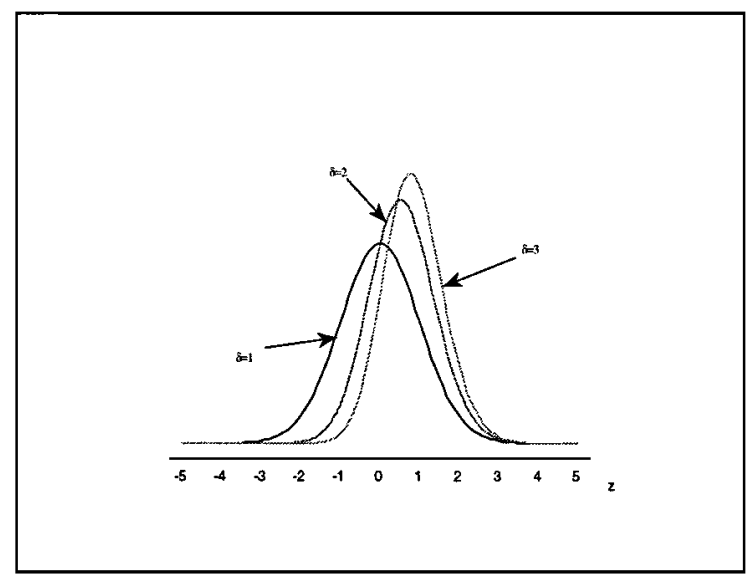

Figure 2. The densities corresponding to LE alternatives $\Phi^{\delta}$ for $\delta=1,2$ and 3 , where $\Phi$ is the standard normal cdf.

It may be noted that as $\delta$ changes from 1 , the mean and the variance of the distribution $G$ are both altered. For illustration, we provide some numerical values from Van der Laan (1970), under the assumption that $F$ corresponds to the standard normal distribution.

\begin{tabular}{|c|c|c|c|}
\hline$\delta$ & 1 & 2 & 3 \\
\hline Mean of $G$ & 0 & $\frac{1}{\sqrt{\pi}}=0.56$ & $\frac{3}{2 \sqrt{\pi}}=0.85$ \\
\hline Variance of $G$ & 1 & $1-\frac{1}{\pi}=0.68$ & $1-\frac{9-2 \sqrt{3}}{4 \pi}=0.56$ \\
\hline
\end{tabular}

Remark 9: A variation of the LE alternatives, called the proportional hazards (PH) alternatives is also of interest. The $\mathrm{PH}$ alternatives have been more popularly used in the context of reliability and/or survival analysis. Here the $\operatorname{cdf} F$ and $G$ satisfy the relationship $G=1-(1-F)^{\gamma}$, where $\gamma>0$, is a parameter. Thus the $\mathrm{PH}$ alternatives are, in fact, $\mathrm{LE}$ alternatives defined between the two survival functions, $1-G$ and $1-F$. One example of $\mathrm{PH}$ alternatives is where the underlying distribution is exponential: $F(x)=1-e^{-\mu x}$ and $G(x)=1-e^{-\eta x}$, so that the $\mathrm{PH}$ alternatives hold with $\gamma=\eta / \mu$. It can be shown that under the $\mathrm{PH}$ alternatives, the ratio of the hazard functions corresponding to $G$ and $F$ is a constant, which is a reasonable assumption in some SPC applications.

To illustrate the run length distribution calculations, we computed $P(N \leq k)$ for several values of $k$ for a given two-sided precedence chart. We considered three cases for which the in-control ARL 
is between 500 and 600 (see Table 1). These are: $1 . m=100, n=25, j=13, P_{0}=0.99 ; 2 . m=100, n$ $=11, j=6, P_{0}=0.995 ; 3 . m=500, n=25, j=13, P_{0}=0.9973$. In all three cases we used the normal shift and the LE alternative. Since we have for the LE alternative $\psi(u)=u^{\delta}$ and for the PH alternative $\psi(u)=1-(1-u)^{\delta}$, we can use a substitution argument to show that (see Theorem 5 in the Appendix), in the case of the median, the run length probabilities under the $\mathrm{PH}$ alternative are equal to the corresponding ones for the LE alternative. The results for the LE alternative are shown in Table 3. This table also shows probabilities for the in-control case which are of course the probabilities of a false alarm. Since the precedence chart is distribution-free, these probabilities do not depend on $F$ and $G$.

We observe that the control charts 1 and 3 are much better in detecting departures from the incontrol case than chart 2 . For example, we observe that for case $2 P(N \leq 10)=0.376$ if the alternative is normal and the shift $\theta=0.5$, whereas for case 1 and 3 we have $P(N \leq 10)=0.736$ and $P(N \leq 10)=$ 0.744 , respectively. This is not surprising because of the larger values of $m$ and/or $n$. The more or less similar behavior of charts 1 and 3 is explained by the larger value of $m$ for chart 3 , which increases the run length probabilities of chart 3 , and the larger value of $P_{0}$ for chart 3 , which decreases the run length probabilities of chart 3.

\subsection{2 $\quad A R L$}

Using equations (16) and (17) and Theorem 2, the $A R L$ of the proposed charts can be evaluated under various alternatives. For example, under the normal shift alternatives $\psi(u)=\Phi\left(\Phi^{-1}(u)-\theta\right)$ from Theorem 2, whereas under the LE and the PH alternatives, $\psi(u)$ equals $u^{\delta}, \delta>0$ and $1-(1-u)^{\gamma}, \gamma>0$, respectively. These expressions, in turn, yield the respective out-of-control $A R L$ expressions. 
Table 3. Cumulative run length probabilities $P(N \leq k)$ under alternatives $^{1}$

\begin{tabular}{|c|c|c|c|c|c|c|c|c|}
\hline \multirow[b]{2}{*}{$k$} & \multirow[b]{2}{*}{$F=G$} & \multicolumn{4}{|c|}{ Normal } & \multicolumn{3}{|c|}{ Lehmann, $G=F^{\delta}$} \\
\hline & & $\theta=.25$ & $\theta=.5$ & $\theta=1$ & $\theta=1.5$ & $\delta=1.5$ & $\delta=2$ & $\delta=3$ \\
\hline \multirow{3}{*}{1} & 0.008 & 0.038 & 0.186 & 0.807 & 0.996 & 0.053 & 0.200 & 0.614 \\
\hline & 0.004 & 0.013 & 0.054 & 0.358 & 0.810 & 0.014 & 0.041 & 0.154 \\
\hline & 0.003 & 0.020 & 0.141 & 0.810 & 0.998 & 0.027 & 0.141 & 0.591 \\
\hline \multirow{3}{*}{2} & 0.016 & 0.073 & 0.320 & 0.943 & 1 & 0.099 & 0.333 & 0.796 \\
\hline & 0.009 & 0.026 & 0.103 & 0.566 & 0.951 & 0.027 & 0.079 & 0.269 \\
\hline & 0.005 & 0.040 & 0.259 & 0.959 & 1 & 0.054 & 0.258 & 0.816 \\
\hline \multirow{3}{*}{5} & 0.038 & 0.160 & 0.557 & 0.995 & 1 & 0.208 & 0.556 & 0.937 \\
\hline & 0.022 & 0.063 & 0.226 & 0.833 & 0.997 & 0.063 & 0.174 & 0.488 \\
\hline & 0.013 & 0.096 & 0.514 & 0.999 & 1 & 0.126 & 0.507 & 0.975 \\
\hline \multirow{3}{*}{10} & 0.073 & 0.269 & 0.736 & 0.999 & 1 & 0.330 & 0.719 & 0.979 \\
\hline & 0.043 & 0.117 & 0.376 & 0.947 & 1 & 0.117 & 0.293 & 0.669 \\
\hline & 0.025 & 0.179 & 0.744 & 1 & 1 & 0.231 & 0.729 & 0.998 \\
\hline \multirow{3}{*}{25} & 0.160 & 0.463 & 0.898 & 1 & 1 & 0.527 & 0.872 & 0.996 \\
\hline & 0.101 & 0.246 & 0.620 & 0.994 & 1 & 0.240 & 0.503 & 0.855 \\
\hline & 0.060 & 0.375 & 0.947 & 1 & 1 & 0.456 & 0.934 & 1 \\
\hline \multirow{3}{*}{50} & 0.269 & 0.621 & 0.959 & 1 & 1 & 0.672 & 0.938 & 0.999 \\
\hline & 0.183 & 0.392 & 0.787 & 0.999 & 1 & 0.376 & 0.668 & 0.934 \\
\hline & 0.117 & 0.581 & 0.993 & 1 & 1 & 0.667 & 0.987 & 1 \\
\hline \multirow{3}{*}{100} & 0.416 & 0.760 & 0.985 & 1 & 1 & 0.792 & 0.972 & 1 \\
\hline & 0.311 & 0.563 & 0.901 & 1 & 1 & 0.535 & 0.805 & 0.974 \\
\hline & 0.217 & 0.786 & 1 & 1 & 1 & 0.847 & 0.999 & 1 \\
\hline \multirow{3}{*}{500} & 0.785 & 0.947 & 0.999 & 1 & 1 & 0.947 & 0.996 & 1 \\
\hline & 0.720 & 0.866 & 0.991 & 1 & 1 & 0.847 & 0.964 & 0.998 \\
\hline & 0.661 & 0.990 & 1 & 1 & 1 & 0.994 & 1 & 1 \\
\hline \multirow{3}{*}{1000} & 0.890 & 0.977 & 1 & 1 & 1 & 0.974 & 0.999 & 1 \\
\hline & 0.860 & 0.952 & 0.998 & 1 & 1 & 0.922 & 0.985 & 0.999 \\
\hline & 0.855 & 0.999 & 1 & 1 & 1 & 0.999 & 1 & 1 \\
\hline
\end{tabular}

${ }^{1}$ Case 1. (top row of each cell) $m=100, n=25, j=13, P_{0}=0.99, A R L_{0}=510.8$;

Case 2. (middle row of each cell) $m=100, n=11, j=6, P_{0}=0.995, A R L_{0}=574.5$;

Case 3. (bottom row of each cell) $m=500, n=25, j=13, P_{0}=0.9973, A R L_{0}=526.2$. 
Again, for example, under the normal shift alternatives, the out-of-control $A R L$ of the proposed two-sided precedence chart is given by

$$
A R L_{N S}=\int_{00}^{1} \int_{0}^{t}[1-F(s, t, j, n, \theta)]^{-1} \frac{m !}{(a-1) !(b-a-1) !(m-b) !} s^{a-1}(t-s)^{b-a-1}(1-t)^{m-b} d s d t
$$

where

$$
F(s, t, j, n, \theta)=\frac{1}{B(j, n-j+1)} \sum_{h=0}^{n-j} \frac{(-1)^{h}}{j+h}\left(\begin{array}{c}
n-j \\
h
\end{array}\right)\left[\left\{\Phi\left(\Phi^{-1}(t)-\theta\right)\right\}^{j+h}-\left\{\Phi\left(\Phi^{-1}(s)-\theta\right)\right\}^{j+h}\right] .
$$

For the LE alternative we have the following theorem, which is similar to Theorem 1.

\section{Theorem 3}

In case of the Lehmann alternative $\psi(s)=s^{\delta}$ (w.l.o.g. take $\delta \leq 1$ ), we have

(a) For the one-sided chart, $A R L_{\delta}<\infty$ iff $m-b-(n-j)>0$.

(b) For the two-sided chart, $A R L_{\delta}<\infty$ iff $(a / \delta)(n-j+1)+j(m-b+1)-j(n-j+1)>0$.

Proof: The proof is given in the Appendix.

The combination of Theorem 1 and Theorem 4 implies that for the two-sided case it is possible to design a chart with an infinite $A R L_{0}$ and a finite $A R L_{\delta}$, whereas this is impossible for the one-sided case.

We discuss the in-control and the out-of-control $A R L$ values in the context of robustness of control charts in the next section.

\subsubsection{Robustness}

One of the nice properties of NP control charts is that the in-control $A R L$ does not depend on the underlying process distribution. However, the normal theory based (in general parametric) charts are often highly affected by a change in the underlying distribution. Since precedence charts are based on test samples of size $n$, we compare these charts with parametric charts based on subgroup size $n$. 
Amin, Reynolds and Bakir (1995) show that the in-control $A R L$ of the normal-based $\bar{X}$ chart, based on subgroup size $n=10$, depends very much on the underlying distribution. For heavy-tailed distributions the false alarm rates may become unacceptably high. In their case, when the underlying distribution is exponential (or Gamma(1,1)), the false alarm rate is 5 times higher than under normality. To examine the in-control $A R L$-sensitivity of other standard charts such as the CUSUM and the EWMA, for subgroup sizes larger than 1, we examined several situations. We restrict ourselves to subgroup size $n=5$. Therefore, a shift of $l \sigma$ corresponds to a shift of $\sqrt{5} l \sigma_{\bar{x}}$.

We first choose the parameters of the parametric charts such that they are good charts to detect either a small or a large shift when normality is fulfilled. Using programs ANYARL and ANYGETH (Hawkins and Olwell, 1998; Chapter 10), we found that when the underlying distribution is normal, the optimal values of the chart parameters, $(h, k)$, are $(4.61, \sqrt{5} * 0.5 / 2)=(4.61,0.56)$ and $(h, k)=$ $(0.86, \sqrt{5} * 2 / 2)=(0.86,2.24)$, respectively, when shifts of $\sigma / 2$ and $2 \sigma$ units are to be detected with an in-control $A R L$ of 500 and a subgroup size $n=5$. For the EWMA charts, chart parameters $(L, \lambda)=$ $(3.054,0.4)$ and $(L, \lambda)=(2.814,0.1)$ are good values to detect shifts of approximately $2 \sigma\left(\approx 4.4 \sigma_{\bar{x}}\right)$ and $\sigma / 2\left(\approx 1.1 \sigma_{\bar{x}}\right)$ units, respectively, under the same conditions as for the CUSUM charts (see for example, Montgomery, 1997; p. 338).

In order to examine the in-control $A R L$-robustness of these charts, we computed the in-control $A R L$ 's for these charts based on the normal distribution with mean $\mu$ and variance $\sigma^{2}$, for some nonnormal distributions, but with the same mean and the same variance as that of the normal distribution. As for non-normal distributions, the $t$ and the Laplace distributions were used to investigate the effect of heavy tails, the uniform distribution was used to study the effect of light tails and the gamma distributions were used to examine the possible effect of skewness. In addition, to study the effect of 
a change in the process variance, we computed the $A R L$ 's for these charts based on the normal $(0,1)$ distribution and for the normal $(0,1.1)$ distribution. The results are shown in Table 4.

The conclusions drawn from the above table are clear. The CUSUM in-control $A R L$ is very sensitive to the departures from normality and for a change of the variance, especially when it is designed to detect a large shift. The EWMA chart, on the other hand, is quite robust against such departures from normality, especially when $\lambda$ is small. This conclusion is also drawn in Borror, Montgomery and Runger (1999) for EWMA charts for individuals. The EWMA chart, however, does not appear to be robust against a change of the variance.

Table 4. In-control $A R L$ values for several distributions

\begin{tabular}{|c|c|c|c|c|c|}
\hline & PREC & \multicolumn{2}{|c|}{ CUSUM } & \multicolumn{2}{|c|}{ EWMA } \\
\hline Distribution & $\begin{array}{l}m=1,000 \\
n=5, j=3 \\
P_{0}=0.9978 \\
a=48, b=953\end{array}$ & $\begin{array}{l}h=4.61 \\
k=0.56 \\
n=5\end{array}$ & $\begin{array}{l}H=0.86 \\
K=2.24 \\
n=5\end{array}$ & $\begin{array}{l}L=2.81 \\
\lambda=0.1 \\
n=5\end{array}$ & $\begin{array}{l}L=3.05 \\
\lambda=0.4 \\
n=5\end{array}$ \\
\hline $\mathrm{N}(0,1)$ & 501.89 & 500.00 & 500.00 & 500.00 & 500.00 \\
\hline Laplace $(0,1)$ & 501.89 & 390.64 & 196.18 & 418.77 & 289.82 \\
\hline $\mathrm{U}(0,1)$ & 501.89 & $\overline{549.16}$ & 1820.13 & 494.73 & 703.72 \\
\hline $\mathrm{N}(0,1.1)$ & 501.89 & 206.38 & 196.69 & 214.49 & 199.79 \\
\hline Gamma $(1,1)$ & 501.89 & 310.74 & 121.53 & 404.27 & 207.62 \\
\hline $\operatorname{Gamma}(4,1)$ & 501.89 & 462.43 & 245.68 & 472.33 & 370.20 \\
\hline $\mathrm{t}(4)$ & 501.89 & 266.86 & 131.90 & 380.98 & $190 . \overline{39}$ \\
\hline $\mathrm{t}(40)$ & 501.89 & 456.76 & 413.44 & 470.87 & 380.63 \\
\hline
\end{tabular}

To be able to compare the out-of-control $A R L$ 's, next we computed the $A R L$ 's for three distributions: normal $(0,1), \mathrm{t}(4)$ and $\Gamma(1,1)$. The chart parameters were adjusted in such a way that the 
in-control ARL of each of the charts is (approximately) equal to 500. The results are shown in Tables 5,6 and 7 , respectively.

The conclusions are as follows. The CUSUM and EWMA charts are better for both normal and $\Gamma(1,1)$. For normal, the differences between the precedence chart and parametric charts are not so large for shifts larger than $1.5 \sigma$. This is not surprising since the proposed charts are Shewhart type charts. For $\mathrm{t}(4)$, small shifts are better detected by the CUSUM (with small $k$ ) and the EWMA (with small $\lambda$ ) charts, whereas large shifts are better detected by the precedence charts.

Table 5. $A R L$ values for the normal $\left(\mu, \sigma^{2}\right)$ distribution

\begin{tabular}{|c|c|c|c|c|c|}
\hline & PREC & \multicolumn{2}{|c|}{ CUSUM } & \multicolumn{2}{c|}{ EWMA } \\
\hline & $\begin{array}{c}m=1000 \\
n=5\end{array}$ & $\begin{array}{c}k=4.61 \\
k=0.56 \\
n=5\end{array}$ & $\begin{array}{c}h=0.86 \\
k=2.24 \\
n=5\end{array}$ & $\begin{array}{c}L=2.81 \\
\lambda=0.1 \\
n=5\end{array}$ & $\begin{array}{c}L=3.05 \\
\lambda=0.4 \\
n=5\end{array}$ \\
\hline \hline 0.00 & 501.89 & 500.00 & 500.00 & 500.00 & 500.00 \\
\hline 0.25 & 240.93 & 33.35 & 162.72 & 25.75 & 56.05 \\
\hline 0.50 & 71.70 & 8.78 & 36.09 & 8.86 & 11.00 \\
\hline 0.75 & 24.22 & 4.86 & 10.60 & 5.33 & 4.76 \\
\hline 1.00 & 9.79 & 3.40 & 4.21 & 3.87 & 2.97 \\
\hline 1.50 & 2.70 & 2.22 & 1.56 & 2.57 & 1.77 \\
\hline 2.00 & 1.37 & 1.77 & 1.09 & 2.04 & 1.25 \\
\hline 2.50 & 1.07 & 1.34 & 1.01 & 1.81 & 1.04 \\
\hline 3.00 & 1.01 & 1.06 & 1.00 & 1.40 & 1.00 \\
\hline 4.00 & 1.00 & 1.00 & 1.00 & 1.01 & 1.00 \\
\hline
\end{tabular}


Table 6. $A R L$ values for the $\mathrm{t}(4)$ distribution

\begin{tabular}{|c|c|c|c|c|c|}
\hline & PREC & \multicolumn{2}{|c|}{ CUSUM } & \multicolumn{2}{c|}{ EWMA } \\
\hline & $\begin{array}{c}m=1000 \\
\text { Shift } \sigma\end{array}$ & $\begin{array}{c}h=5.38 \\
k=0.56 \\
n=5\end{array}$ & $\begin{array}{c}h=1.81 \\
k=2.24 \\
n=5\end{array}$ & $\begin{array}{c}L=3.04 \\
\lambda=0.1 \\
n=5\end{array}$ & $\begin{array}{c}L=3.65 \\
\lambda=0.4 \\
n=5\end{array}$ \\
\hline 0.00 & 501.89 & 495.23 & 499.47 & 507.91 & 504.44 \\
\hline 0.25 & 300.09 & 41.88 & 340.58 & 28.28 & 128.87 \\
\hline 0.50 & 32.70 & 10.24 & 136.21 & 7.86 & 22.45 \\
\hline 0.75 & 11.16 & 5.54 & 35.50 & 3.98 & 7.11 \\
\hline 1.00 & 2.26 & 3.86 & 9.84 & 2.47 & 3.77 \\
\hline 1.50 & 1.16 & 2.49 & 2.31 & 1.38 & 1.20 \\
\hline 2.00 & 1.02 & 1.98 & 1.34 & 1.06 & 1.03 \\
\hline 2.50 & 1.00 & 1.63 & 1.06 & 1.01 & 1.00 \\
\hline 3.00 & 1.00 & 1.20 & 1.01 & 1.00 & 1.00 \\
\hline 4.00 & 1.00 & 1.01 & 1.00 & 1.00 & 1.00 \\
\hline
\end{tabular}

Table 7. $A R L$ values for the $\operatorname{Gamma}(1,1)$ distribution

\begin{tabular}{|c|c|c|c|c|c|}
\hline & PREC & \multicolumn{2}{|c|}{ CUSUM } & \multicolumn{2}{c|}{ EWMA } \\
\cline { 2 - 6 } Shift/ $\sigma$ & $\begin{array}{c}m=1000 \\
n=5\end{array}$ & $\begin{array}{c}H=5.18 \\
K=0.56 \\
n=5\end{array}$ & $\begin{array}{c}h=1.75 \\
k=2.24 \\
n=5\end{array}$ & $\begin{array}{c}L=2.81 \\
\lambda=0.1 \\
n=5\end{array}$ & $\begin{array}{c}L=3.05 \\
\lambda=0.4 \\
n=5\end{array}$ \\
\hline 0.00 & 501.89 & 496.43 & 498.22 & 509.76 & 505.68 \\
\hline 0.25 & 439.01 & 41.04 & 182.85 & 25.18 & 80.83 \\
\hline 0.50 & 256.45 & 10.28 & 69.94 & 7.66 & 18.73 \\
\hline 0.75 & 125.31 & 5.44 & 24.50 & 3.91 & 6.98 \\
\hline 1.00 & 61.87 & 3.75 & 9.05 & 2.47 & 3.70 \\
\hline 1.50 & 15.81 & 2.43 & 2.32 & 1.38 & 1.76 \\
\hline 2.00 & 4.83 & 1.90 & 1.36 & 1.03 & 1.20 \\
\hline 2.50 & 1.63 & 1.61 & 1.02 & 1.00 & 1.00 \\
\hline 3.00 & 1.02 & 1.15 & 1.00 & 1.00 & 1.00 \\
\hline 4.00 & 1.00 & 1.00 & 1.00 & 1.00 & 1.00 \\
\hline
\end{tabular}




\section{Effect of the reference sample size}

It may be noted that when the in-control process distribution $F$ is known, one can set up a control chart for the test sample quantile $Y_{(j: n)}$, simply by taking $L C L=\xi_{p_{1}}$ and $U C L=\xi_{p_{2}}$, where $\xi_{p_{1}}$ and $\xi_{p_{2}}$ are two specified quantiles of $F$, given $0<p_{1}<p_{2}<1$. The quantity $j$ can be found given $n, p_{1}, p_{2}$ and a specified value of the probability, say, $P\left(\xi_{p_{1}} \leq Y_{(j: n)} \leq \xi_{p_{2}}\right)$, from the binomial tables. However, when $F$ is unknown, as in the present case, the population quantiles can be estimated by taking a reference $\left(X\right.$-) sample from $F$, and using $X_{(a: m)}$ and $X_{(b: m)}$, where $a=\left[m p_{1}\right]+1$ and $b=\left[m p_{2}\right]$ +1 , for the control limits. Using estimated control limits introduces extra variation and additional complications, since the signaling events now become dependent. Since it is well known that for $c=[m p]+1$ and $0<p<1, X_{(c: m)}$ is a consistent estimator of $\xi_{p}$ (the $p$-quantile of $F$ ), as the reference sample size $m$ becomes large, the $X$-order statistics will become close to the corresponding population quantiles. Thus, a natural question is how large should $m$ be so that the extra sampling variation due to the estimation of the limits can be ignored. In other words, given $n, j$ and $0<p_{1}<p_{2}<1$, for what value of $m$ is $P^{(1)}=P\left(\xi_{p_{1}} \leq Y_{(j: n)} \leq \xi_{p_{2}}\right)$ sufficiently "close" to $P^{(2)}=$ $P_{C}\left(X_{(a: m)} \leq Y_{(j: m)} \leq X_{(b: m)}\right) ?$

Under the continuity of $F, P^{(1)}$ can be easily calculated as the difference between two (incomplete-) Beta-integrals

$$
\int_{0}^{p_{2}} f(u ; j, n-j+1) d u-\int_{0}^{p_{1}} f(u ; j, n-j+1) d u,
$$

where $f(u ; j, n-j+1)$ is the density function of the Beta-distribution with parameters $j$ and $n-j+1$. The probability $P^{(2)}$, of course, is simply given by the left-hand side of (3).

The expressions for $P^{(1)}$ and $P^{(2)}$ were evaluated with $n=7, j=4, p_{1}=0.1, p_{2}=0.9$, $n=7, j=3, p_{1}=0.1, p_{2}=0.9$ and $n=25, j=13, p_{1}=0.1, p_{2}=0.7$, for various values of $m$ ranging 
from 100 to 100,000 . These values were chosen so that $P^{(1)}$ resembled what might be a $P_{0}$ value in a typical application. The results are shown in Table 8.

Table 8. Effect of reference sample size on the precedence control chart

\begin{tabular}{|c|c|c|c|c|c|c|c|c|}
\hline$n, j, p_{1}, p_{2}$ & $P^{(1)}$ & $m: 100$ & 500 & 1,000 & $\begin{array}{r}P^{(2)} \\
5,000\end{array}$ & 10,000 & 50,000 & 100,000 \\
\hline $\begin{array}{c}n=7, j=4 \\
p_{1}=0.1, p_{2}=0.9\end{array}$ & 0.994544 & 0.990782 & 0.993871 & 0.994212 & 0.994478 & 0.994511 & 0.994537 & 0.994541 \\
\hline $\begin{array}{c}n=7, j=3 \\
p_{1}=0.1, p_{2}=0.9\end{array}$ & 0.974132 & 0.969874 & 0.973265 & 0.973698 & 0.974045 & 0.974088 & 0.974123 & 0.974128 \\
\hline $\begin{array}{c}n=25, j=13 \\
p_{1}=0.1, p_{2}=0.7\end{array}$ & 0.982530 & 0.965029 & 0.979349 & 0.980964 & 0.982221 & 0.982376 & 0.982499 & 0.982515 \\
\hline
\end{tabular}

An inspection of the entries in the table makes the effect of the size of the reference sample quite clear. For $m=1,000$ or more, the two probabilities are reasonably close, suggesting that the $X$ order statistics are "very close" to their population counterparts, so that one could ignore the dependence caused by estimation of the control limits. Put another way, for $m$ around 1,000, one could approximate the in-control run-length distribution of the proposed charts by the geometric with the probability of a signal equal to $1-P\left(\xi_{p_{1}} \leq Y_{(j \cdot n)} \leq \xi_{p_{2}}\right)$, where $p_{1}=a / m$ and $p_{2}=b / m$. It follows that for such values of $m$, the $A R L_{0}$ is equal to the reciprocal of the probability of a signal. However, for small to moderate values of $m$, exact calculations, using the formulas given earlier, are recommended. 


\section{Operating characteristic $(\mathrm{OC})$ function}

Consider the case of two-sided charts (one-sided charts can be treated similarly). As noted earlier, the properties of the chart depends on the coverage probability $p=p\left(m, n, j ; F, G_{h}\right)$ which is the OC function. For example, the probability of a false alarm is simply $1-p(m, n, j ; F, F)$. In this section we examine the probability $p$ in more detail. The following result is useful to study properties of the chart when the process is not necessarily in-control.

Theorem 4 The probability $p=P\left(X_{(a: m)} \leq Y_{(j: n)} \leq X_{(b: m)}\right)$, where $1 \leq a<b \leq m$, is given by

$$
p=p(m, n, j, F, G)=\sum_{r=a}^{b-1} \frac{\left(\begin{array}{c}
m \\
r
\end{array}\right)}{B(j, n-j+1)} I(r ; m, n, j, F, G),
$$

where

$$
I(r ; m, n, j, F, G)=\int_{0}^{1}\left[F G^{-1}(s)\right]^{r}\left[1-F G^{-1}(s)\right]^{m-r} s^{j-1}(1-s)^{n-j} d s .
$$

Proof: The result follows from the fact that $p=P\left(a \leq W_{j} \leq b-1\right)$, together with a result from $\mathrm{CV}$ (1996) about the distribution of the precedence statistic $W_{j}$.

Remark 10: In the in-control case, the integral $I$ in (19) can be evaluated exactly and this yields $p(m, n, j, F, F)=\frac{\sum_{r=a}^{b-1}\left(\begin{array}{l}m \\ r\end{array}\right) B(r+j, m+n-r-j+1)}{B(j, n-j+1)}$. This expression simplifies to the left hand of (3).

Remark 11: The integral $I(r ; m, n, j)$ in (19) can be re-written as

$$
\int_{-\infty}^{\infty} F^{r}(x)[1-F(x)]^{m-r} G^{j-1}(x)[1-G(x)]^{n-j} d G(x) .
$$

Thus, the properties of the OC function of the proposed charts depend on the underlying process distributions through the composite function $\psi=F G^{-1}$. Applying results of Theorem 4 we now obtain the OC function of the proposed chart to some specific alternatives. First consider the normal shift case. 


\subsection{OC function for normal shift alternatives (NS)}

Assume that $G(\mathrm{t})=\Phi(\mathrm{t}-\theta)$ and $F(t)=\Phi(\mathrm{t})$, where $\Phi$ is the standard normal cdf. Thus $\theta$ represents the shift in the mean. Writing $H=\Phi, \theta_{1}=0, \theta_{2}=\theta$, and applying (a) in Theorem 2 and simplifying, the OC function for the normal shift alternatives is found to be

$$
p_{N S}(m, n, j, \Phi, \theta)=\sum_{r=a}^{b-1} \frac{\left(\begin{array}{c}
m \\
r
\end{array}\right)}{B(j, n-j+1)} \int_{-\infty}^{\infty} \Phi^{r}(t+\theta)[1-\Phi(t+\theta)]^{m-r} \Phi^{j-1}(t)[1-\Phi(t)]^{n-j} d \Phi(t) .
$$

\subsection{OC function for Lehmann alternatives (LE)}

Under Lehmann alternatives, considerable simplification is achieved since here $\psi(s)=s^{1 / \delta}$ and therefore the integral $I(r, m, n, j)$ can be calculated exactly. After some simplifications, the OC function is obtained,

$$
p_{L E}(m, n, j, \delta)=\sum_{r=a}^{h-1} \frac{\left(\begin{array}{c}
m \\
r
\end{array}\right)}{B(j, n-j+1)} \sum_{w=0}^{m-r}(-1)^{w}\left(\begin{array}{c}
m-r \\
w
\end{array}\right) B\left(j+\frac{r+w}{\delta}, n-j+1\right) .
$$

\subsection{OC function for proportional hazards alternatives (PH)}

Under the PH alternatives, as noted in Theorem 2, part (e), we have here $\psi(u)=1-(1-u)^{1 / \delta}$ and the integral $I(r, m, n, j)$ can also be exactly evaluated. After some simplifications, the OC function is obtained

$$
p_{P H}(m, n, j, \gamma)=\sum_{r=a}^{b-1} \frac{\left(\begin{array}{c}
m \\
r
\end{array}\right)}{B(j, n-j+1)} \sum_{w=0}^{r}(-1)^{w}\left(\begin{array}{c}
r \\
w
\end{array}\right) B\left(j, n-j+1+\frac{m+w-r}{\gamma}\right) .
$$

Remark 12: All computations in this paper are done in Mathematica 3.0 (see Wolfram, 1996). We wrote Mathematica notebooks to design precedence charts for given values of $m, n, j$ and $P_{0}$. These 
notebooks are also suitable for computing in- and out-of-control $A R L$-values. The notebooks are available from the third author.

\section{Numerical Example}

Montgomery (1997; Table 6.1) gives a set of data on the inside diameters of piston rings manufactured by a forging process. Twenty-five samples, each of size five, were collected when the process was thought to be in-control. Traditional Shewhart X-bar and $\mathrm{R}$ charts provided no indication of an out-of-control condition, so these "trial" limits were adopted for use in on-line (Phase II) process control. Fifteen additional (test) samples were then collected (Montgomery, 1997; Table 6.2) from the same process and compared against these control charts. For the same data we construct a precedence control chart by treating the whole set of $125(25 \times 5)$ observations as the reference sample. We have $m=125, n=5$ and suppose a false alarm rate of 0.0027 . Using our program we find that the control limits for the future sample median are given by the $5^{\text {th }}$ and the $121^{\text {th }}$ ordered values of the reference sample and this chart guarantees an exact false alarm rate of 0.001866 . The control chart is drawn and the medians of the 15 test samples are plotted on this chart. The plot is shown in Figure 2.

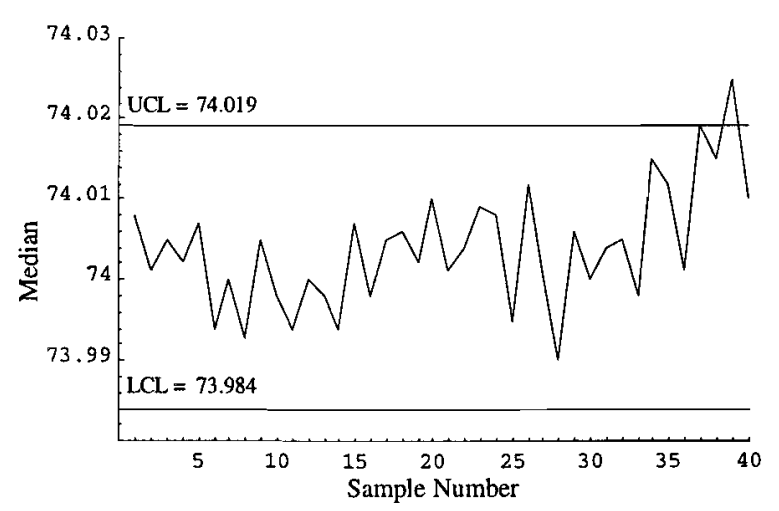

Figure 2. Precedence Control Chart for Piston Ring Data 
It is seen that for the precedence chart the $37^{\text {th }}$ sample was a close call (the control statistic value was equal to the $U C L$ ) and the chart signaled on the $39^{\text {th }}$ sample. The original Shewhart X-bar chart for these data showed all three of the $37^{\text {th }}, 38^{\text {th }}$ and the $39^{\text {th }}$ points above the corresponding $U C L$ and the $40^{\text {th }}$ point almost equal to the $U C L$. However, the X-bar chart had a false alarm rate (at best approximately) equal to 0.0027 , whereas the precedence chart has an exact false alarm rate of 0.0019 . In addition, the calculations needed for the proposed chart are also quite simple. Further, it is not necessary to check on the variance. Thus, it seems that in practice the precedence charts can provide an attractive alternative to the usual parametric charts.

\section{Acknowledgements}

This research was supported in part by awards from the Philips Corporation, The Netherlands and the College of Commerce and Business Administration, the University of Alabama.

\section{References}

Amin, R., Reynolds, M.R., Jr., and Bakir, S. (1995), "Nonparametric quality control charts based on the sign test," Commun. Statist.-Theor. Meth., 24, 1597-1624.

Borror, C.M., Montgomery, D.C., and Runger, G.C. (1999), "Robustness of the EWMA control chart to non-normality," J. Qual. Technol., 31, 309-316.

Chakraborti, S. (2000), "Run length, average run length and false alarm rate of Shewhart X-bar chart: exact derivations by conditioning." Commun. Statist.-Simula. Computa., 29, 61-81.

Chakraborti, S. and Van der Laan, P. (1996), "Precedence tests and confidence bounds for complete data: an overview and some results," The Statistician, 45, 351-369.

Chakraborti, S. and Van der Laan, P. (1997), "An overview of precedence tests for censored data," Biometrical J., 39, 99-116.

Chakraborti, S., Van der Laan, P., and Bakir, S. T. (2000), "Nonparamertric Process Control: an overview and some results," J. Qual. Technol., accepted.

Gibbons, J. D. and Chakraborti, S. (1992), Nonparametric Statistical Inference, $3^{\text {rd }}$ edn., Marcel Dekker, New York. 
Hawkins, D.M. and Olwell, D.H. (1998), "Cumulative Sum Charts and Charting for Quality Improvement," Springer, New York.

Janacek, G. J. and Meikle, S. E. (1997), "Control charts based on medians," The Statistician, 46, 19-31.

Lehmann, E. L. (1953), “ The power of rank tests,” Ann. Math. Statist., 24, 23-43.

Mathisen, H. C. (1943), "A method of testing the hypothesis that two samples are from the same population," Ann. Math. Statist., 14, 188-194.

Montgomery, D. C. (1997), "Introduction to Statistical Quality Control," $3^{\text {rd }}$ ed., John Wiley, New York.

Nelson, L. S. (1963), “Tables for a precedece life test," Technometrics, 5, 491-499.

Nelson, L. S. (1993), “Tests on early failures—the precedence life test," J. Qual. Technol., 25, 140149.

Quesenberry, C. P. (1997), "SPC Methods for Quality Improvement," John Wiley, New York.

Van der Laan, P. (1970), "Simple distribution-free confidence intervals for a difference in location," $\mathrm{Ph}$. D. thesis, Eindhoven University of Technology.

Wolfram, S. (1996), "The Mathematica Book," $3^{\text {rd }}$ ed., Cambridge University Press, Cambridge.

Woodall, W. H. (2000), "Controversies and contradictions in statistical process control," (with discussion). J. Qual. Technol. Forthcoming.

Woodall, W. H. and Montgomery, D. C. (1999), "Research issues and ideas in statistical process control," J. Qual. Technol. 31, 376-386.

\section{APPENDIX}

\section{Theorem 1}

(a) For the one-sided control chart,

$$
A R L_{0}<\infty \Leftrightarrow m-b-(n-j)>0
$$

(b) For the two-sided control chart,

$$
A R L_{0}<\infty \Leftrightarrow a(n-j+1)+j(m-b+1)-j(n-j+1)>0 .
$$

Theorem 3 In case of the Lehmann alternative $G F^{-1}(s)=s^{\delta}$ (w.l.o.g. take $\delta \leq 1$ ), we have 
(b) For the one-sided control chart,

$$
A R L_{\delta}<\infty \Leftrightarrow m-b-(n-j)>0
$$

(b) For the two-sided control chart,

$$
A R L_{\delta}<\infty \Leftrightarrow(a / \delta)(n-j+1)+j(m-b+1)-j(n-j+1)>0 .
$$

We first prove Theorem 1 part (b) and show that, using some results of this proof, the proof of part (a) is straightforward. Some additional techniques are needed to prove Theorem 3.

\section{Proof of Theorem 1 (part b)}

Define the constants $c=\frac{m !}{(a-1) !((b-a-1) !(m-b) !}$ and $\beta=B(j, n-j+1)$. Since $A R L_{0}=\sum_{k=0}^{\infty} D(k)$, we have for the two-sided chart :

$$
A R L_{0}=c \int_{0}^{1} \int_{0}^{t} \frac{s^{a-1}(t-s)^{b-a-1}(1-t)^{m-b}}{g(s, 1-t)} d s d t=c \int_{0}^{1} \int_{0}^{1-t} \frac{s^{a-1}(1-t-s)^{b-a-1} t^{m-b}}{g(s, t)} d s d t,
$$

where

*1 $g(s, t)=g_{1}(s)+g_{2}(t)$,

where

*2

$$
g_{1}(s)=\beta^{-1} \sum_{h=0}^{n-j}(-1)^{h}\left(\begin{array}{c}
n-j \\
h
\end{array}\right) s^{j+h}(j+h)^{-1} \text { and }
$$

$$
g_{2}(t)=1-\beta^{-1} \sum_{h=0}^{n-j}(-1)^{h}\left(\begin{array}{c}
n-j \\
h
\end{array}\right)(1-t)^{j+h}(j+h)^{-1} .
$$

Then

$$
g_{1}{ }^{\prime}(s)=\beta^{-1} \sum_{h=0}^{n-j}(-1)^{h}\left(\begin{array}{c}
n-j \\
h
\end{array}\right) s^{j+h-1}=\beta^{-1} s^{j-1} \sum_{h=0}^{n-j}(-s)^{h}\left(\begin{array}{c}
n-j \\
h
\end{array}\right)=\beta^{-1} s^{j-1}(1-s)^{n-j},
$$

where we use Newton's binomial formula for the last equality. Similarly, we have

$$
g_{2}^{\prime}(t)=\beta^{-1} t^{n-j}(1-t)^{j-1}
$$

Now using integration by parts, iteratively, we get equivalent formulas for $g_{1}(s)$ and $g_{2}(t)$. These are convenient to work with in the sequel. 
$* 3$

$$
\begin{aligned}
g_{1}(s) & =\int \beta^{-1} s^{j-1}(1-s)^{n-j} d s=(\beta j)^{-1} \int(1-s)^{n-j} d s{ }^{j} \\
& =(\beta j)^{-1} s^{j}(1-s)^{n-j}+(\beta j)^{-1}(n-j) \int s^{j}(1-s)^{n-j-1} d s+C_{1} \\
& =\ldots \ldots \ldots=(\beta j)^{-1} s^{j}(1-s)^{n-j}+(\beta j)^{-1} s^{j} P_{1}(s)+\beta^{-1} c_{1} \int s^{n-1} d s+C_{2} \\
& =s^{j}\left((\beta j)^{-1}(1-s)^{n-j}+P_{2}(s)\right)+C,
\end{aligned}
$$

where $P_{1}(s)$ and $P_{2}(s)$ are polynomials with at least one root in $s, c_{1}$ is a constant and $C, C_{1}, C_{2}$ are (sums of) integration constants. We know that $g_{1}(0)=0$ which implies that $C=0$. Similarly, we have

$* 4 \quad g_{2}(t)=t^{n-j+1}\left((\beta(n-j+1))^{-1}(1-t)^{j-1}+P_{3}(t)\right)$

where $P_{3}(t)$ is a polynomial with at least one root in $\mathrm{t}$. Since $g_{1}{ }^{\prime}(s)>0$ and $\mathrm{g}_{2}{ }^{\prime}(\mathrm{t})>0$ for $0<t<1,0<s<1-t$ $g(s, t)$ is strictly increasing in $s$ and $t$ within the region of integration. Therefore, the only root of $g(s, t)$ is $(0,0)$ and hence the only possible singularity of the integrand of $A R L_{0}$ is at $(0,0)$. We now investigate how the integrand behaves in the neighborhood of $(0,0)$. To this end, divide the integration region $G$ into two parts:

$$
G=\{(s, t) \mid 0 \leq t \leq 1,0 \leq s \leq 1-t\}=H \cup(G \backslash H),
$$

where

$$
H=\left\{(s, t) \mid 0 \leq s, 0 \leq t, 0 \leq s^{j}+t^{n-j+1} \leq(1 / 2)^{j(n-j+1)}\right\} .
$$

The factor $(1 / 2)^{\mathrm{j}(\mathrm{n}-\mathrm{j}+1)}$ is used to make sure that $H$ is entirely contained in $G$. Then,

$$
A R L_{0}=c \iint_{G \backslash H} \frac{s^{a-1}(1-t-s)^{b-a-1} t^{m-b}}{g(s, t)} d s d t+c \iint_{H} \frac{s^{a-1}(1-t-s)^{b-a-1} t^{m-b}}{g(s, t)} d s d t=c A+c B
$$

Since $(0,0)$ is the only possible singularity in the integrand and the integration region of $\mathrm{A}$ is finite we know that $A<\infty$. Therefore,

*5 $A R L_{0}<\infty \Leftrightarrow B<\infty$.

To examine the behavior of the integrand near $(0,0)$ we transform the integral using the following coordinates: *6

$$
s=(r \cos x)^{n-j+1} \text { and } t=(r \sin x)^{j} .
$$

Under this transformation, the integration region becomes:

$$
H=\left\{(r, x) \mid 0 \leq x \leq \pi / 2,0 \leq r^{j(n-j+1)} \leq(1 / 2)^{j(n-j+1)}\left((\cos x)^{j(n-j+1)}+(\sin x)^{j(n-j+1)}\right)^{-1}\right\}
$$


To simplify this integration region we again split it into two parts. The observation that for

$$
u=\min _{x \in[0, \pi / 2]}\left((\cos x)^{j(n-j+1)}+(\sin x)^{j(n-j+1)}\right)^{-1}
$$

we have $0<u<\infty$, leads us to $H=J \cup(H / J)$ with

*7 $J=\{(r, x) \mid 0 \leq x \leq \pi / 2,0 \leq r \leq U\}, \quad U=\frac{1}{2} u^{1 / j(n-j+1)}$.

Since the only possible singularity in the original integration region is $(s, t)=(0,0)$, a singularity in the transformed region can only occur for $r=0$. Therefore, integration over $H / J$ is finite and when we define $C$ similar to $B$, but with integration region $H$ replaced by $J$, we have

$* 8 \quad B<\infty \Leftrightarrow C<\infty$.

Recall from calculus that when we want to use transformed coordinates we have to multiply the integrand with the Jacobian

$$
\begin{aligned}
\left|\frac{\partial(s, t)}{\partial(r, x)}\right| & =\left|\begin{array}{ll}
\frac{\partial(r \cos x)^{n-j+1}}{\partial r} & \frac{\partial(r \cos x)^{n-j+1}}{\partial x} \\
\frac{\partial(r \sin x)^{j}}{\partial r} & \frac{\partial(r \sin x)^{j}}{\partial x}
\end{array}\right| \\
& =\left|\begin{array}{ll}
(n-j+1) r^{n-j}(\cos x)^{n-j+1}-(n-j+1) r^{n-j+1}(\cos x)^{n-j} \sin x \\
j r^{j-1}(\sin x)^{j} & j r^{j}(\sin x)^{j-1} \cos x
\end{array}\right| \\
& \left.=j(n-j+1) r^{n}(\cos x)^{n-j+2}(\sin x)^{j-1}+(\cos x)^{n-j}(\sin x)^{j+1}\right) \\
& =j(n-j+1) r^{n}(\cos x)^{n-j}(\sin x)^{j-1}
\end{aligned}
$$

Then using $* 1, * 3, * 4, * 6$ and $* 7$ we have

$$
\begin{aligned}
C & =\iint_{J} \frac{s^{a-1}(1-t-s)^{b-a-1} t^{m-b}}{g(s, t)} d s d t= \\
& =\lim _{\delta \downarrow 0} \int_{r=\delta}^{U} \int_{x=0}^{\pi / 2} \frac{(r \cos x)^{(n-j+1)(a-1)}\left(1-(r \sin x)^{j}-(r \cos x)^{n-j+1}\right)^{b-a-1}(r \sin x)^{j(m-b)}}{g(r \sin x, r \cos x)}\left|\frac{\partial(s, t)}{\partial(r, x)}\right| d x d r \\
& =\lim _{\delta \downarrow_{0}} \int_{r=\delta}^{U} \int_{x=0}^{\pi / 2} \frac{r^{(n-j+1)(a-1)+j(m-b)+n} h_{1}(r, x)}{r^{j(n-j+1)} h_{2}(r, x)} d x d r
\end{aligned}
$$

where 


$$
\begin{aligned}
h_{1}(r, x) & =j(n-j+1)(\cos x)^{(n-j+1) a-1}\left(1-(r \sin x)^{j}-(r \cos x)^{n-j+1}\right)^{b-a-1}(\sin x)^{j(m-b+1)-1}, \\
h_{2}(r, x) & =(\cos x)^{j(n-j+1)}(\beta j)^{-1}\left(1-(r \cos x)^{n-j+1}\right)^{n-j}+P_{2}\left((r \cos x)^{n-j+1}\right) \\
& +(\sin x)^{j(n-j+1)}(\beta(n-j+1))^{-1}\left(1-(r \sin x)^{j}\right)^{j-1}+P_{3}\left((r \cos x)^{j}\right) .
\end{aligned}
$$

Let us take a closer look at $h_{2}(r, x)$. For the original problem we know that the only possible singularity was at $(s, t)=(0,0)$. Therefore, we use $* 6$ to observe that

*9 $\quad r>0 \Rightarrow h_{2}(r, x)>0$

Moreover, we have for all $x \in[0, \pi / 2]$ :

$* 10 \quad h_{2}(0, x)=(\cos x)^{j(n-j+1)}(\beta j)^{-1}+(\sin x)^{j(n-j+1)}\left(\beta(n-j+1)^{-1}>0\right.$.

Let us now consider the roots of $h_{1}(r, x)$. Clearly, these roots are $x=0$ and $x=\pi / 2$. In fact,

$* 11 \quad x \neq 0$ and $x \neq \pi / 2 \Rightarrow h_{1}(r, x)>0$.

To exclude these points we split $[0, \pi / 2]=[\Delta, \pi / 2-\Delta] \cup E_{\Delta}$ with $0<\Delta<\pi / 2$ and $E_{\Delta}=[0, \pi / 2] /[\Delta, \pi / 2-\Delta]$.

Then,

$$
C=\lim _{\delta \downarrow} \int_{r=\delta}^{U} \int_{x=\Delta}^{\pi / 2-\Delta} \frac{r^{(n-j+1)(a-1)+j(m-b)+n} h_{1}(r, x)}{r^{j(n-j+1)} h_{2}(r, x)} d x d r+\lim _{\delta \downarrow 0} \int_{r=\delta}^{U} \int_{E_{\Delta}} \frac{r^{(n-j+1)(a-1)+j(m-b)+n} h_{1}(r, x)}{r^{j(n-j+1)} h_{2}(r, x)} d x d r=D+E .
$$

Since $D<\infty \Rightarrow E<\infty$ we have

*12 $C<\infty \Leftrightarrow D<\infty$.

Now because of $* 9, * 10$ and $* 11$ there exist $M_{1}>0$ and $M_{2}<\infty$ such that

$$
M_{1} \leq \frac{h_{1}(r, x)}{h_{2}(r, x)} \leq M_{2} \quad \text { for all } 0 \leq r \leq U \text { and } \Delta \leq \mathrm{x} \leq \pi / 2-\Delta
$$

Therefore,

$$
\begin{aligned}
& \lim _{\delta \downarrow} \int_{r=\delta}^{U} \int_{x=\Delta}^{\pi / 2-\Delta} \frac{r^{(n-j+1)(a-1)+j(m-b)+n}}{r^{j(n-j+1)}} M_{1} d x d r \leq C \leq \lim _{\delta \downarrow 0} \int_{r=\delta}^{U} \int_{x=\Delta}^{\pi / 2-\Delta} \frac{r^{(n-j+1)(a-1)+j(m-b)+n}}{r^{j(n-j+1)}} M_{2} d x d r \\
& \Leftrightarrow \lim _{\delta \downarrow 0} \int_{r=\delta}^{U} \frac{r^{(n-j+1)(a-1)+j(m-b)+n}}{r^{j(n-j+1)}} M_{1}\left(\frac{\pi}{2}-2 \Delta\right) d r \leq C \leq \lim _{\delta \downarrow 0} \int_{r=\delta}^{U} \frac{r^{(n-j+1)(a-1)+j(m-b)+n}}{r^{j(n-j+1)}} M_{2}\left(\frac{\pi}{2}-2 \Delta\right) d r .
\end{aligned}
$$

Since 
*13 $\lim _{\delta \downarrow 0} \int_{r=\delta}^{U} r^{\alpha} d r<\infty \Leftrightarrow \alpha>-1$,

we use the bounds to see that

$* 14$

$$
\begin{aligned}
C<\infty & \Leftrightarrow(n-j+1)(a-1)+j(m-b)+n-j(n-j+1)>-1 \\
& \Leftrightarrow a(n-j+1)+j(m-b+1)-j(n-j+1)>0 .
\end{aligned}
$$

Combination of $* 5, * 8, * 12$ and $* 14$ completes the proof.

Q.E.D.

\section{Proof of Theorem 1 (part a)}

For the one-sided case we have

$$
A R L_{0}=\int_{0}^{1} \frac{t^{b-1}(1-t)^{m-b}}{g_{2}(1-t)} d t=\int_{0}^{1} \frac{(1-t)^{b-1} t^{m-b}}{g_{2}(t)} d t
$$

Therefore, the results $* 4$ and $* 13$ can be used to finish the proof.

Note that the transformation of the integration variables $(* 6)$ and corresponding integrating regions is not only of theoretical importance. We found that the implementation of the numerical integrals converges faster and is more stable when we use the transformed variables and region to deal with the area near the singularity.

\section{Proof of Theorem 3 (part b)}

Let $\lambda=1 / \delta$ and assume without loss of generality $\lambda \geq 1$. Then, we use $* 1$ and $* 2$ with $s$ and $t$ replaced by $G F^{-1}(s)=s^{1 / \lambda}$ and $G F^{-1}(t)=t^{1 / \lambda}$, respectively, to obtain

$$
\begin{aligned}
A R L_{\delta} & =\int_{0}^{1} \int_{0}^{t} \frac{s^{a-1}(t-s)^{b-a-1}(1-t)^{m-b}}{g\left(s^{1 / \lambda}, 1-t^{1 / \lambda}\right)} d s d t=\int_{0}^{1} \int_{0}^{t^{\lambda}} \frac{s^{\lambda(a-1)}\left(t^{\lambda}-s^{\lambda}\right)^{b-a-1}\left(1-t^{\lambda}\right)^{m-b} \lambda^{2}(s t)^{\lambda-1}}{g(s, 1-t)} d s d t, \\
& =\int_{0}^{1} \int_{0}^{(1-t)^{\lambda}} \frac{s^{\lambda a-1}\left((1-t)^{\lambda}-s^{\lambda}\right)^{b-a-1}\left(1-(1-t)^{\lambda}\right)^{m-b} \lambda^{2}(1-t)^{\lambda-1}}{g(s, t)} d s d t .
\end{aligned}
$$

Again we have to deal with a possible singularity in $(s, t)=(0,0)$. Let us now investigate the behavior of $f(t, \lambda)=\left(1-(1-t)^{\lambda}\right)^{m-b}$ for $t \approx 0$. First note that $f(t,\lfloor\lambda] \leq f(t, \lambda) \leq f(t,[\lambda\rceil$. However, for each integer $k>0$, we have $f(t, k)=\left(1-(1-t)^{k}\right)^{m-b}=a t^{m-b}+O\left(t^{m-b+1}\right)$, where $a$ is a constant. Therefore, we can always find constants $\mathrm{c}>0$ and $\mathrm{d}>0$ such that $c t^{m-b} \leq f(t, \lambda) \leq d t^{m-b}$ for $t$ close to 0 . This basically means that to prove the 
theorem we may replace $\left(1-(1-t)^{\lambda}\right)^{m-b}$ by $t^{m-b}$. The rest of the proof is completely analogous to the proof of Theorem 1, part b.

Q.E.D.

\section{Theorem 3, part a, can be proved similarly.}

Theorem 5: In the median case we have $P_{P H}(N=k)=P_{L E}(N=k)$.

Proof:

From equation (8) we observe that it suffices to show that $D_{P H}^{*}(k)=D_{L E}^{*}(k)$ for $k=1,2, \ldots$

Let $f(s, t)=\frac{m !}{(a-1) !(b-a-1) !(m-b) !} s^{a-1}(t-s)^{b-a-1}(1-t)^{m-b}$.

Using equation (9) we have

$$
\begin{aligned}
D_{L E}^{*}(k) & =E_{X_{(a: m)}, X_{(v: m)}}\left(p^{k}\right)=\int_{0}^{1} \int_{0}^{t}\left[P\left\{G F^{-1}(s) \leq U_{(j ; n)} \leq G F^{-1}(t)\right\}\right]^{k} f(s, t) d s d t \\
& =\int_{0}^{1} \int_{0}^{t}\left[P\left\{s^{\delta} \leq U_{(j: n)} \leq t^{\delta}\right\}\right]^{k} f(s, t) d s d t \\
& =\int_{0}^{1} \int_{v}^{1}\left[P\left\{(1-u)^{\delta} \leq U_{(j: n)} \leq(1-v)^{\delta}\right\}\right]^{k} f(1-u, 1-v) d u d v \\
& =\int_{0}^{1} \int_{v}^{1}\left[P\left\{1-(1-v)^{\delta} \leq 1-U_{(j ; n)} \leq 1-(1-u)^{\delta}\right\}\right]^{k} f(1-u, 1-v) d u d v \\
& =\int_{0}^{1} \int_{0}^{u}\left[P\left\{1-(1-v)^{\delta} \leq U_{(j: n)} \leq 1-(1-u)^{\delta}\right\}\right]^{k} f(u, v) d v d u \\
& =D_{P H}^{*}(k),
\end{aligned}
$$

where we use the fact that $U_{(j: n)} \sim 1-U_{(j: n)}$, because $j$ is the median and we use the definition of $f$ and the fact that $a-1=m-b$ for the median case to observe that $f(v, u)=f(I-u, I-v)$. 